\title{
Bonsai Trees, or How to Delegate a Lattice Basis
}

\author{
David Cash $^{1, \star}$, Dennis Hofheinz ${ }^{2, \star \star}$, Eike Kiltz $^{3, \star \star \star}$, and Chris Peikert ${ }^{4, \dagger}$ \\ ${ }^{1}$ University of California, San Diego \\ cdc@ucsd.edu \\ 2 Karlsruhe Institute of Technology \\ Dennis.Hofheinz@kit.edu \\ 3 Cryptology \& Information Security Group, CWI, Amsterdam, The Netherlands \\ kiltz@cwi.nl \\ ${ }^{4}$ Georgia Institute of Technology \\ cpeikertacc.gatech.edu
}

\begin{abstract}
We introduce a new lattice-based cryptographic structure called a bonsai tree, and use it to resolve some important open problems in the area. Applications of bonsai trees include:

- An efficient, stateless 'hash-and-sign' signature scheme in the standard model (i.e., no random oracles), and

- The first hierarchical identity-based encryption (HIBE) scheme (also in the standard model) that does not rely on bilinear pairings.

Interestingly, the abstract properties of bonsai trees seem to have no known realization in conventional number-theoretic cryptography.
\end{abstract}

\section{Introduction}

Lattice-based cryptographic schemes have undergone rapid development in recent years, and are attractive due to their low asymptotic complexity and potential resistance to quantum-computing attacks. One notable recent work in this area is due to Gentry, Peikert, and Vaikuntanathan [25], who constructed an efficient 'hash-and-sign' signature scheme and an identity-based encryption (IBE) scheme. (IBE is a powerful cryptographic primitive in which any string can serve as a public key [53].)

Abstractly, the GPV schemes are structurally quite similar to Rabin/Rabin-Williams signatures [50] (based on integer factorization) and the Cocks/Boneh-Gentry-Hamburg IBEs [18, 13] (based on the quadratic residuosity problem), in that they all employ a so-called "preimage sampleable" trapdoor function as a basic primitive. As a result,

* Part of work performed while at Georgia Institute of Technology.

${ }^{\star \star}$ Part of work performed while at CWI and supported by an NWO Veni grant.

$\star \star \star$ Supported by the research program Sentinels.

$\dagger$ This material is based upon work supported by the National Science Foundation under Grants CNS-0716786 and CNS-0749931, and by the US Department of Homeland Security under Contract Number HSHQDC-07-C-00006. Any opinions, findings, and conclusions or recommendations expressed in this material are those of the author(s) and do not necessarily reflect the views of the National Science Foundation or the US Department of Homeland Security. 
they have so far required the random oracle model (or similar heuristics) for their security analysis. This is both a theoretical drawback and also a practical concern (see, e.g., [35]), so avoiding such heuristics is an important goal.

Another intriguing open question is whether any of these IBE schemes can be extended to deliver richer levels of functionality, as has been done in pairing-based cryptography since the work of Boneh and Franklin [12]. For example, the more general notion of hierarchical IBE [33, 26] permits multiple levels of secret-key authorities. This notion is more appropriate than standard IBE for large organizations, can isolate damage in the case of secret-key exposure, and has further applications such as forwardsecure encryption [16] and broadcast encryption [21, 58].

\subsection{Our Results}

We put forward a new cryptographic notion called a bonsai tree, and give a realization based on hard lattice problems. (Section 1.2 gives an intuitive overview of bonsai trees, and Section 1.4 discusses their relation to other primitives and techniques.) We then show that bonsai trees resolve some central open questions in lattice-based cryptography: to summarize, they remove the need for random oracles in many important applications, and facilitate delegation for purposes such as hierarchical IBE.

Our first application of bonsai trees is an efficient, stateless signature scheme that is secure in the standard model (no random oracles) under conventional lattice assumptions. Our scheme has a 'hash-and-sign' flavor that does not use the key-refresh/ authentication-tree paradigm of many prior constructions (both generic [28, 43] and specialized to lattice assumptions [37]), and in particular it does not require the signer to keep any state. (Statelessness is a crucial property in many real-world scenarios, where distinct systems may sign relative to the same public key.) In our scheme, the verification key, signature length, and verification time are all an $O(k)$ factor larger than in the random-oracle scheme of [25], where $k$ is the output length of a chameleon hash function, and the $O(\cdot)$ notation hides only a 1 or 2 factor. The signing algorithm is essentially as efficient as the one from [25] 1 The underlying hard problem is the standard short integer solution (SIS) problem dating back to the seminal work of Ajtai [5], which is known to be as hard as several worst-case approximation problems on lattices (see also [41,25]). Via SIS, the security of our signature scheme rests upon the hardness of approximating worst-case problems on $n$-dimensional lattices to within an $\tilde{O}\left(\sqrt{k} \cdot n^{3 / 2}\right)$ factor; this is only a $\sqrt{k}$ factor looser than that of [25].

Our second application is a collection of various hierarchical identity-based encryption (HIBE) schemes, which are the first HIBEs that do not rely on bilinear pairings. Our main scheme works in the standard model, also making it the first non-pairingbased IBE (hierarchical or not) that does not use random oracles or qualitatively similar heuristics. The underlying hard problem is the standard learning with errors (LWE) problem as defined by Regev, which may be seen as the 'dual' of SIS and is also as hard as certain worst-case lattice problems [51, 45]; LWE is also the foundation for the plain IBE of [25], among many other recent cryptographic schemes.

\footnotetext{
${ }^{1}$ Our signing algorithm performs about $k$ forward computations of a trapdoor function, plus one inversion (which dominates the running time).
} 
Additionally, our HIBE is anonymous across all levels of the hierarchy, i.e., a ciphertext conceals (computationally) the identity to which is was encrypted. Anonymity is a useful property in many applications, such as fully private communication [7] and searching on encrypted data [11,1]. While there are a few anonymous (non-hierarchical) IBEs [12, 20, 13, 25], only one other HIBE is known to be anonymous [15].

\subsection{Overview of Bonsai Trees and Applications}

The ancient art of bonsai is centered around a tree and the selective control thereof by an arborist, the tree's cultivator and caretaker. By combining natural, undirected growth with controlled propagation techniques such as wiring and pruning, arborists cultivate trees according to a variety of aesthetic forms.

Similarly, cryptographic bonsai is not so much a precise definition as a collection of principles and techniques, which can be employed in a variety of ways. (The informal description here is developed technically in Section 3.) The first principle is the tree itself, which in our setting is a hierarchy of trapdoor functions having certain properties. The arborist can be any of several entities in the system - e.g., the signer in a signature scheme or a simulator in a security proof - and it can exploit both kinds of growth, undirected and controlled. Briefly stated, undirected growth of a branch means that the arborist has no privileged information about the associated function, whereas the arborist controls a branch if it knows a trapdoor for the function. Moreover, control automatically extends down the hierarchy, i.e., knowing a trapdoor for a parent function implies knowing a trapdoor for any of its children.

In our concrete lattice-based instantiation, the functions in the tree are indexed by a hierarchy of public lattices chosen at random from a certain 'hard' family (i.e., one having a connection to worst-case problems). The lattices may be specified by a variety of means, e.g., a public key, interaction via a protocol, a random oracle, etc. Their key property is that they naturally form a hierarchy as follows: every lattice in the tree (excepting the root) is a higher-dimensional superlattice of its parent. Specifically, a parent lattice in $\mathbb{R}^{m}$ is simply the restriction of its child(ren) in $\mathbb{R}^{m^{\prime}}$ (where $m^{\prime}>$ $m$ ) to the first $m$ dimensions. As we shall see shortly, this hierarchical relationship means that a parent lattice naturally 'subsumes' its children (and more generally, all its descendants).

Undirected growth in our realization is technically straightforward, emerging naturally from the underlying hard average-case lattice problems (SIS and LWE). This growth is useful primarily for letting a simulator embed a challenge problem into one or more branches of the tree (but it may have other uses as well).

To explain controlled growth, we first need a small amount of technical background. As explored in prior works on lattice-based cryptography (e.g., [27, 30, 29, 25, 49, 45]), a lattice has a 'master trapdoor' in the form of a short basis, i.e., a basis made up of relatively short lattice vectors. Knowledge of such a trapdoor makes it easy to solve a host of seemingly hard problems relative to the lattice, such as decoding within a bounded distance, or randomly sampling short lattice vectors. The reader may view a short basis for a lattice as roughly analogous to the factorization of an integer, though we emphasize that there are in general many distinct short bases that convey roughly 'equal power' with respect to the lattice. 
In light of the above, we say that an arborist controls a branch of a bonsai tree if it knows a short basis for the associated lattice. The hierarchy of lattices is specially designed so that any short basis of a parent lattice can be easily extended to a short basis of any higher-dimensional child lattice, with no loss in quality. This means that control of a branch implicitly comes with control over all its offshoots. In a typical application, the privileged entity in the system (e.g., the signer in a signature scheme) will know a short basis for the root lattice, thus giving it control over the entire tree. Other entities, such as an attacker, will generally have less power, though in some applications they might even be given control over entire subtrees.

So far, we have deliberately avoided the question of how an arborist comes to control a (sub)tree by acquiring a short basis for the associated lattice. A similar issue arises in other recent cryptographic schemes [25, 49, 45], but in a simpler setting involving only a single lattice and short basis (not a hierarchy). In these schemes, one directly applies a special algorithm, originally conceived by Ajtai [4] and recently improved by Alwen and Peikert [6], which generates a hard random lattice together with a short basis 'from scratch.' At first glance, the algorithms of [4, 6] seem useful only for controlling a new tree entirely by its root, which is not helpful if we need finer-grained control. Fortunately, we observe that the same technique used for extending an already-controlled lattice also allows us to 'graft' a solitary controlled lattice onto an uncontrolled branch 2

This whole collection of techniques, therefore, allows an arborist to achieve a primary bonsai aesthetic: a carefully controlled tree that nonetheless gives the appearance of having grown without any outside intervention. As we shall see next, bonsai techniques can reduce the construction of complex cryptographic schemes to the design of simple combinatorial games between an arborist and an adversary.

Application 1: Hash-and-Sign without Random Oracles. Our end goal is a signature scheme that meets the de facto notion of security, namely, existential unforgeability under adaptive chosen-message attack [28]. By a standard, efficient transformation using chameleon hashes [34] (which have efficient realizations under conventional lattice assumptions, as we show), it suffices to construct a weakly secure scheme, namely, one that is existentially unforgeable under a static attack in which the adversary nonadaptively makes all its queries before seeing the public key.

Our weakly secure scheme signs messages of length $k$, the output length of the chameleon hash. The public key represents a binary bonsai tree $T$ of depth $k$ in a compact way, which we describe in a moment. The secret key is a short basis for the lattice $\Lambda_{\varepsilon}$ at the root of the tree, which gives the signer control over all of $T$. To sign a string $\mu \in\{0,1\}^{k}$ (which is the chameleon hash of the 'true' message $m$ ), the signer first derives the lattice $\Lambda_{\mu}$ from $T$ by walking the root-to-leaf path specified by $\mu$. The signature is simply a short nonzero vector $\mathbf{v} \in \Lambda_{\mu}$, chosen at random from the 'canonical' Gaussian distribution (which can be sampled efficiently using the signer's control over $\Lambda_{\mu}$ ).

\footnotetext{
${ }^{2}$ It is worth noting that in [4, 6], even the simple goal of generating a solitary lattice together with a short basis actually proceeds in two steps: first start with a sufficient amount of random undirected growth, then produce a single controlled offshoot by way of a certain linear algebraic technique. Fittingly, this is analogous to the traditional bonsai practice of growing a new specimen from a cutting of an existing tree, which is generally preferred to growing a new plant 'from scratch' with seeds.
} 
A verifier can check the signature $\mathbf{v}$ simply by deriving $\Lambda_{\mu}$ itself from the public key, and checking that $\mathbf{v}$ is a sufficiently short nonzero vector in $\Lambda_{\mu}$.

The bonsai tree $T$ is represented compactly by the public key in the following way. First, the root lattice $\Lambda_{\varepsilon}$ is specified completely. Then, for each level $i=0, \ldots, k-1$, the public key includes two blocks of randomness that specify how a parent lattice at level $i$ branches into its two child lattices. We emphasize that all nodes at a given depth use the same two blocks of randomness to derive their children.

The proof of security is at heart a combinatorial game on the tree between the simulator $\mathcal{S}$ and forger $\mathcal{F}$, which goes roughly as follows. The forger gives the simulator a set $M=\left\{\mu_{1}, \ldots, \mu_{Q}\right\}$ of messages, and $\mathcal{S}$ needs to cultivate a bonsai tree (represented by $p k$ ) so that it controls some set of subtrees that cover all of $M$, yet is unlikely to control the leaf of whatever arbitrary message $\mu^{*} \notin M$ that $\mathcal{F}$ eventually produces as a forgery. If the latter condition happens to hold true, then the forger has found a short nonzero vector in an uncontrolled lattice, in violation of the underlying assumption.

To satisfy these conflicting constraints, $\mathcal{S}$ colors red all the edges on the root-to-leaf paths of the messages in $M$, and lets all the other edges implicitly be colored blue. The result is a forest of at most $Q \cdot k$ distinct blue subtrees $\left\{B_{\ell}\right\}$, each growing off of some red path by a single blue edge. The simulator chooses one of these subtrees $B_{\ell}$ uniformly at random (without regard to its size), guessing that the eventual forgery will lie in $B_{\ell}$. It then cultivates a bonsai tree so that all the growth on the path up to and throughout $B_{\ell}$ is undirected (by embedding its given challenge instance as usual), while all the remaining growth in $T \backslash B_{\ell}$ is controlled. This goal can be achieved within the confines of the public key by controlling one branch at each level leading up to $B_{\ell}$ (namely, the branch growing off of the path to $B_{\ell}$ ), and none thereafter.

Application 2: Hierarchical Identity-Based Encryption. Bonsai trees also provide a very natural and flexible approach for realizing HIBE. For simplicity, consider an authority hierarchy that is a binary tree, which suffices for forward-secure encryption and general HIBE itself [16]. The master public key of the scheme describes a binary bonsai tree, which mirrors the authority hierarchy. The root authority starts out by controlling the entire tree, i.e., it knows a trapdoor short basis for the lattice at the root. Each authority is entitled to control its corresponding branch of the tree. Any entity in the hierarchy can delegate control over an offshoot branch to the corresponding sub-authority, simply by computing and revealing a short basis of the associated child lattice. In this framework, encryption and decryption algorithms based on the LWE problem are standard.

For the security proof, the simulator again prepares a bonsai tree so that it controls certain branches (which should cover the adversary's queries), while allowing the undirected growth of others (corresponding to the adversary's target identity). This can be accomplished in a few ways, with different advantages and drawbacks in terms of the security notion achieved and the tightness of the reduction. One notion is security against a selective-identity attack, where the adversary must declare its target identity before seeing the public key, but may adaptively query secret keys afterward. In this model, the simulator can cultivate a bonsai tree whose growth toward the (known) target identity is undirected, while controlling each branch off of that path; this setup makes it easy for the simulator to answer any legal secret-key query. 
A stronger notion is a fully adaptive attack, where the adversary may choose its target identity after making its secret-key queries. There are generic combinatorial techniques for converting selective-identity-secure (H)IBE schemes into fully secure ones; we show how to apply and optimize these techniques to our HIBE. First, we use the techniques of Boneh and Boyen [8] construct a fully secure HIBE scheme in the random oracle model. The basic idea is to hash all identities; this way, the target identity can be dynamically embedded as the answer to a random oracle query. Secondly, we demonstrate that other tools of Boneh and Boyen [9] can be adapted to our setting to yield a fully secure HIBE scheme without random oracles. This works by hashing identities to branches of a bonsai tree, where a probabilistic argument guarantees that any given identity hashes to a controlled branch with a certain probability. We can adjust this probability in the right way, so that with non-negligible probability, all queried identities hash to controlled branches, while the target identity hashes to an uncontrolled branch. In our probabilistic argument, we employ admissible hash functions (AHFs), as introduced by [9]. However, as we will explain in Section 5.4 their original AHF definition and proof strategy do not take into consideration the statistical dependence of certain crucial events. We circumvent this with a different AHF definition and a different proof.

Based on the above description, the reader may still wonder whether secret-key delegation is actually secure, i.e., whether the real and simulated bases are drawn from the same probability distribution. In fact, they may not be! For example, under the most straightforward method of extending a basis, the child basis actually contains the parent basis as a submatrix, so it is clearly insecure to reveal the child. We address this issue with an additional bonsai principle of randomizing control, using the 'oblivious' Gaussian sampling algorithm of [25]. This produces a new basis under a 'canonical' distribution, regardless of the original input basis, which ensures that the real system and simulation coincide. The randomization increases the length of the basis by a small factor - which accumulates geometrically with each delegation from parent to child - but for reasonable depths, the resulting bases are still short enough to be useful when all the parameters are set appropriately. (See Section 1.3 for more details.)

For achieving security under chosen-ciphertext attacks (CCA security), a transformation due to Boneh, Canetti, Halevi, and Katz [10] gives a CCA-secure HIBE for depth $d$ from any chosen plaintext-secure HIBE for depth $d+1$. Alternatively, we observe that the public and secret keys in our HIBE scheme are of exactly the same 'type' as those in the recent CCA-secure cryptosystem of [45], so we can simply plug that scheme into our bonsai tree/HIBE framework. Interestingly, the two approaches result in essentially identical schemes.

Variations. This paper focuses almost entirely on bonsai trees that are related, via worst- to average-case reductions, to general lattices. Probably the main drawback is that the resulting public and secret keys are rather large. For example, the public key in our signature scheme is larger by a factor of $k$ (the output length of a chameleon hash function) than that of its random-oracle analogue [25], which is already at least quadratic in the security parameter. Fortunately, the principles of bonsai trees may be applied equally well using analogous hard problems and tools for cyclic/ideal lattices (developed in, e.g., [39, 47, 36, 48, 55, 38]). This approach can 'miniaturize' the bonsai 
trees and most of their associated operations by about a linear factor in the security parameter. The resulting schemes are still not suitable for practice, but their asymptotic behavior is attractive.

\subsection{Complexity and Open Problems}

Here we discuss some additional quantitative details of our schemes, and describe some areas for further research.

Several important quantities in our bonsai tree constructions and applications depend upon the depth of the tree. The dimension of a lattice in the tree grows linearly with its depth, and the size of the trapdoor basis grows roughly quadratically with the dimension.

Accordingly, in our HIBE schemes, the dimension of a ciphertext vector grows (at least) linearly with the depth of the identity to which it is encrypted. Moreover, the (Euclidean) length of an user's trapdoor basis increases geometrically with its depth in the tree (more precisely, with the length of the delegation chain), due to the basis randomization that is performed with each delegation. To ensure correct decryption, the inverse noise parameter $1 / \alpha$ in the associated LWE problem, and hence the approximation factor of the underlying worst-case lattice problems, must grow with the basis length. In particular, a hierarchy of depth $d$ corresponds (roughly) to an $n^{d / 2}$ approximation factor for worst-case lattice problems, where $n$ is the dimension. Because lattice problems are conjectured to be hard to approximate to within even subexponential factors, the scheme may remain secure for depths as large as $d=n^{c}$, where $c<1$.

Our HIBE scheme that enjoys security under a full adaptive-identity attack requires large keys and has a somewhat loose security reduction. In particular, the attack simulation partitions an (implicit) bonsai tree into controlled and undirected branches. This is done in the hope that all user secret key queries refer to controlled branches (so the simulation can derive the corresponding secret key), and that the target identity refers to an undirected branch (so the attack can be converted into one on the LWE problem). This simulation approach (dubbed 'partitioning strategy' in [57]) involves, to a certain extent, guessing the adversary's user secret key and challenge queries. The result is a rather loose security reduction.

In contrast, recent works have achieved tight reductions (and even small keys, in some cases) for pairing-based (H)IBEs under various assumptions [23, 24, 57], and a variant of the GPV IBE (in the random oracle model) also has a tight reduction, but their approaches do not seem to translate to our setting. The issue, essentially, is that our simulator is required to produce a 'master trapdoor' for each queried identity, which makes it difficult to embed the challenge problem into the adversary's view. In prior systems with tight reductions, secret keys are less 'powerful,' so the simulator can embed a challenge while still producing secret keys for any identity (even the targeted one).

A final very interesting (and challenging) question is whether bonsai trees can be instantiated based on other mathematical foundations, e.g., integer factorization. At a very fundamental level, our lattice-based construction seems to rely upon a kind of random self-reducibility that the factorization problem is not known to enjoy. 


\subsection{Related Techniques and Works}

This paper represents a combination of two concurrent and independent works by the first three authors [17] and the fourth author [44], which contained some overlapping results and were accepted to Eurocrypt 2010 under the condition that they be merged.

The abstract properties of bonsai trees appear to have no known realization in conventional number-theoretic cryptography. However, our applications use combinatorial techniques that are similar to those from prior works.

The analysis of our signature scheme is reminiscent of (and influenced by) the recent RSA-based signatures of Hohenberger and Waters [32], but there are some notable structural differences. Most significantly, our scheme does not implicitly 'sign' every prefix of the message as in [32]. Additionally, in contrast with prior hash-andsign schemes based on RSA [22, 19, 31, 32], our simulator cannot use an 'accumulator' to produce signatures for exactly the queried messages, but instead sets up the public key so that it knows enough trapdoors to cover all the messages (and potentially many others). This requires the simulator to cultivate a tree whose structure crucially depends on the global properties of the entire query set, thus inducing the forest of subtrees as described in Section 1.2

The structure of our HIBE is also similar, at a combinatorial level at least, to that of prior pairing-based HIBEs, in that the simulator can 'control' certain edges of an (implicit) tree by choosing certain random exponents itself. However, there are no trapdoor functions per se in pairing-based constructions; instead, the pairing is used to facilitate secret agreement between the encrypter and decrypter. Our approach, therefore, may be seen as a blending of pairing-based techniques and the trapdoor techniques found in [18, 13, 25].

Following the initial dissemination of our results in [17, 44], several extensions and additional applications have been found. Rückert [52] modified our signature scheme to make it strongly unforgeable, and constructed hierarchical identity-based signatures. Agrawal and Boyen [3] constructed a standard-model IBE based on LWE, which is secure under a selective-identity attack; their construction has structure similar to ours, but it does not address delegation, nor does it give an efficient signature scheme. Agrawal, Boneh, and Boyen [2] improved the efficiency of our (H)IBE schemes (under a somewhat stronger LWE assumption), and Boyen [14] used similar techniques to obtain shorter signatures (under a stronger SIS assumption).

\section{Preliminaries}

\subsection{Notation}

For a positive integer $k,[k]$ denotes the set $\{1, \ldots, k\}$; [0] is the empty set. We denote the set of integers modulo an integer $q \geq 1$ by $\mathbb{Z}_{q}$. For a string $x$ over some alphabet, $|x|$ denotes the length of $x$. We say that a function in $n$ is negligible, written negl $(n)$, if it vanishes faster than the inverse of any polynomial in $n$.

The statistical distance between two distributions $\mathcal{X}$ and $\mathcal{Y}$ (or two random variables having those distributions), viewed as functions over a countable domain $D$, is defined as $\max _{A \subseteq D}|\mathcal{X}(A)-\mathcal{Y}(A)|$. 
Column vectors are named by lower-case bold letters (e.g., $\mathbf{x}$ ) and matrices by uppercase bold letters (e.g., $\mathbf{X}$ ). We identify a matrix $\mathbf{X}$ with the ordered set $\left\{\mathbf{x}_{j}\right\}$ of its column vectors, and let $\mathbf{X} \| \mathbf{X}^{\prime}$ denote the (ordered) concatenation of the sets $\mathbf{X}, \mathbf{X}^{\prime}$. For a set $\mathbf{X}$ of real vectors, we define $\|\mathbf{X}\|=\max _{j}\left\|\mathbf{x}_{j}\right\|$, where $\|\cdot\|$ denotes the Euclidean norm.

For any (ordered) set $\mathbf{S}=\left\{\mathbf{s}_{1}, \ldots, \mathbf{s}_{k}\right\} \subset \mathbb{R}^{m}$ of linearly independent vectors, let $\widetilde{\mathbf{S}}=\left\{\widetilde{\mathbf{s}_{1}}, \ldots, \widetilde{\mathbf{s}_{k}}\right\}$ denote its Gram-Schmidt orthogonalization, defined iteratively as follows: $\widetilde{\mathbf{s}_{1}}=\mathbf{s}_{1}$, and for each $i=2, \ldots, k$, the vector $\widetilde{\mathbf{s}_{i}}$ is the component of $\mathbf{s}_{i}$ orthogonal to $\operatorname{span}\left(\mathbf{s}_{1}, \ldots, \mathbf{s}_{i-1}\right)$. In matrix notation, there is a unique $\mathrm{QR}$ decomposition $\mathbf{S}=\mathbf{Q R}$ where the columns of $\mathbf{Q} \in \mathbb{R}^{m \times k}$ are orthonormal (i.e., $\mathbf{Q}^{t} \mathbf{Q}=\mathbf{I} \in \mathbb{R}^{k \times k}$ ) and $\mathbf{R} \in \mathbb{R}^{k \times k}$ is right-triangular with positive diagonal entries; the Gram-Schmidt orthogonalization is $\widetilde{\mathbf{S}}=\mathbf{Q} \cdot \operatorname{diag}\left(r_{1,1}, \ldots, r_{k, k}\right)$. Clearly, $\left\|\widetilde{\mathbf{s}_{i}}\right\| \leq\left\|\mathbf{s}_{i}\right\|$ for all $i$.

\subsection{Cryptographic Definitions}

The main cryptographic security parameter through the paper is $n$, and all algorithms (including the adversary) are implicitly given the security parameter $n$ in unary.

For a (possibly interactive) algorithm $\mathcal{A}$, we define its distinguishing advantage between two distributions $\mathcal{X}$ and $\mathcal{Y}$ to be $|\operatorname{Pr}[\mathcal{A}(\mathcal{X})=1]-\operatorname{Pr}[\mathcal{A}(\mathcal{Y})=1]|$. We use the general notation $\operatorname{Adv}_{\mathrm{SCH}}^{\text {atk }}(\mathcal{A})$ to describe the advantage of an adversary $\mathcal{A}$ mounting an atk attack on a cryptographic scheme $\mathrm{SCH}$, where the definition of advantage is specified as part of the attack. Similarly, we write $\operatorname{Adv}_{\text {PRов }}(\mathcal{A})$ for the advantage of an adversary $\mathcal{A}$ against a computational problem PROB (where again the meaning of advantage is part of the problem definition).

Chameleon hash functions. Chameleon hashing was introduced by Krawczyk and Rabin [34]. For our purposes, we need a slight generalization in the spirit of "preimage sampleable" (trapdoor) functions [25].

A family of chameleon hash functions is a collection $\mathcal{H}=\left\{h_{i}: \mathcal{M} \times \mathcal{R} \rightarrow \mathcal{Y}\right\}$ of functions $h_{i}$ mapping a message $m \in \mathcal{M}$ and randomness $r \in \mathcal{R}$ to a range $\mathcal{Y}$. The randomness space $\mathcal{R}$ is endowed with some efficiently sampleable distribution (which may not be uniform). A function $h_{i}$ is efficiently computable given its description, and the family has the property that for any $m \in \mathcal{M}$, for $h_{i} \leftarrow \mathcal{H}$ and $r \leftarrow \mathcal{R}$, the pair $\left(h_{i}, h_{i}(m, r)\right)$ is uniform over $(\mathcal{H}, \mathcal{Y})$ (up to negligible statistical distance). The chameleon property is that a random $h_{i} \leftarrow \mathcal{H}$ may be generated together with a trapdoor $t$, such that for any output $y \in \mathcal{Y}$ and message $m \in \mathcal{M}$, it is possible (using $t$ ) to efficiently sample $r \in \mathcal{R}$ (under the $\mathcal{R}$ 's distribution) conditioned on the requirement that $h_{i}(m, r)=y$. Finally, the family has the standard collisionresistance property, i.e., given $h_{i} \leftarrow \mathcal{H}$ it should be hard for an adversary to find distinct $(m, r),\left(m^{\prime}, r^{\prime}\right) \in \mathcal{M} \times \mathcal{R}$ such that $h_{i}(m, r)=h_{i}\left(m^{\prime}, r^{\prime}\right)$.

A realization under conventional lattice assumptions of chameleon hash functions (in the above sense) for $\mathcal{M}=\{0,1\}^{\ell}$ is straightforward, using the particular preimage sampleable functions (PSFs) from [25]. Briefly, the chameleon hash function is simply a PSF applied to $m \| r$, which may also be viewed as the sum of two independent PSFs applied to $m$ and $r$, respectively. We omit the details. 
Signatures. A signature scheme SIG for a message space $\mathcal{M}$ is a tuple of PPT algorithms as follows:

- Gen outputs a verification key $v k$ and a signing key $s k$.

- Sign $(s k, \mu)$, given a signing key $s k$ and a message $\mu \in \mathcal{M}$, outputs a signature $\sigma \in\{0,1\}^{*}$.

- $\operatorname{Ver}(v k, \mu, \sigma)$, given a verification key $v k$, a message $\mu$, and a signature $\sigma$, either accepts or rejects.

The correctness requirement is: for all $\mu \in \mathcal{M}$, all possible $(v k, s k) \leftarrow$ Gen and $\sigma \leftarrow$ $\operatorname{Sign}(s k, \mu)$, we have that $\operatorname{Ver}(v k, \mu, \sigma)$ accepts with overwhelming probability (over all the randomness in the experiment).

We recall two standard notions of security for signatures. The first, existential unforgeability under static chosen-message attack, or eu-scma security, is defined as follows: first, the forger $\mathcal{F}$ outputs a list of query messages $\mu_{1}, \ldots, \mu_{Q}$ for some $Q$. Next, $(v k, s k) \leftarrow$ Gen and $\sigma_{i} \leftarrow \operatorname{Sign}\left(s k, \mu_{i}\right)$ are generated for each $i \in[Q]$, then $v k$ and $\sigma_{i}$ (for each $i \in[Q]$ ) are given to $\mathcal{F}$. Finally, $\mathcal{F}$ outputs an attempted forgery $\left(\mu^{*}, \sigma^{*}\right)$. The advantage $\mathcal{A}_{\text {SIG }}^{\text {eu-scma }}(\mathcal{F})$ of $\mathcal{F}$ is the probability that $\operatorname{Ver}\left(v k, \mu^{*}, \sigma^{*}\right)$ accepts and $\mu^{*} \neq \mu_{i}$ for all $i \in[Q]$, taken over all the randomness of the experiment.

Another notion, called existential unforgeability under adaptive chosen-message attack, or eu-acma security, is defined similarly, except that $\mathcal{F}$ is first given $v k$ and may adaptively choose the messages $\mu_{i}$.

Using a family of chameleon hash functions (as defined above), there is a generic construction of eu-acma-secure signatures from eu-scma-secure signatures; e.g., [34]. Furthermore, the construction results in an online/offline signature scheme; see [54]. The basic idea behind the construction is that the signer chameleon hashes the message to be signed, then signs the hashed message using the eu-scma-secure scheme (and includes the randomness used in the chameleon hash with the final signature).

Key-Encapsulation Mechanism (KEM). We present all of our encryption schemes in the framework of key encapsulation, which simplifies the definitions and leads to more modular constructions. A KEM for keys of length $\ell=\ell(n)$ is a triple of PPT algorithms as follows:

- KEM.Gen outputs a public key $p k$ and a secret key $s k$.

- KEM.Encaps $(p k)$ outputs a key $\kappa \in\{0,1\}^{\ell}$ and its encapsulation as $\sigma \in\{0,1\}^{*}$.

- KEM.Decaps $(s k, \sigma)$ outputs a key $\kappa$.

The correctness requirement is: for all possible $(p k, s k) \leftarrow$ KEM.Gen and $(\kappa, \sigma) \leftarrow$ $\operatorname{KEM} . \operatorname{Encaps}(p k)$, we have that $\operatorname{KEM} . \operatorname{Decaps}(s k, \sigma)$ outputs $\kappa$ with all but negl $(n)$ probability.

In this work we are mainly concerned with indistinguishability under chosen-plaintext attack, or ind-cpa security. The attack is defined as: generate $(p k, s k) \leftarrow$ KEM.Gen, $\left(\kappa^{*}, \sigma^{*}\right) \leftarrow \operatorname{KEM}$.Encaps $(p k)$, and $\kappa^{\prime} \leftarrow\{0,1\}^{\ell}$ (chosen uniformly and independently of the other values). The advantage $\operatorname{Adv}_{\text {KEM }}^{\text {ind-cpa }}(\mathcal{A})$ of an adversary $\mathcal{A}$ is its distinguishing advantage between $\left(p k, \sigma^{*}, \kappa^{*}\right)$ and $\left(p k, \sigma^{*}, \kappa^{\prime}\right)$. 
Hierarchical Identity-Based Encryption (HIBE) and Binary Tree Encryption (BTE). In HIBE, identities are strings over some alphabet $\mathcal{I D}$; BTE is the special case of HIBE with identity space $\mathcal{I D}=\{0,1\}$. A HIBE is a tuple of PPT algorithms as follows:

- Setup $\left(1^{d}\right)$ outputs a master public key $m p k$ and root-level user secret key $u s k_{\varepsilon}$. (In the following, $1^{d}$ and $m p k$ are implicit parameters to every algorithm, and every $u s k_{i d}$ is assumed to include $i d$ itself.)

- Extract $\left(u s k_{i d}, i d^{\prime}\right)$, given an user secret key for identity $i d \in \mathcal{I D}^{<d}$ that is a prefix of $i d^{\prime} \in \mathcal{I D}^{\leq d}$, outputs a user secret key $u s k_{i d^{\prime}}$ for identity $i d^{\prime}$.

- Encaps $(i d)$ outputs a key $\kappa \in\{0,1\}^{\ell}$ and its encapsulation as $\sigma \in\{0,1\}^{*}$, to identity $i d$.

- Decaps $\left(u s k_{i d}, \sigma\right)$ outputs a key $\kappa$.

The correctness requirement is: for any identity $i d \in \mathcal{I D}^{\leq d}$, generate $\left(m p k, u s k_{\varepsilon}\right) \leftarrow$ Setup $\left(1^{d}\right), u s k_{i d}$ via any legal sequence of calls to Extract starting from $u s k_{\varepsilon}$, and $(\kappa, \sigma) \leftarrow \operatorname{Encaps}(i d)$. Then $\operatorname{Decaps}\left(u s k_{i d}, \sigma\right)$ should output $\kappa$ with all but negl $(n)$ probability (over all the randomness in the experiment).

There are several attack notions for HIBE. We are mainly concerned with the simple notion of indistinguishability under a chosen-plaintext, selective-identity attack, or sid-ind-cpa security. The attack is defined as follows: first, the adversary $\mathcal{A}$ is given $1^{d}$ and names a target identity $i d^{*} \in \mathcal{I D}^{\leq d}$. Next, $(m p k, m s k) \leftarrow \operatorname{Setup}\left(1^{d}\right),\left(\kappa, \sigma^{*}\right) \leftarrow$ $\operatorname{Encaps}\left(i d^{*}\right)$, and $\kappa^{\prime} \leftarrow\{0,1\}^{\ell}$ are generated. Then $\mathcal{A}$ is given $\left(m p k, \kappa^{*}, \sigma^{*}\right)$, where $\kappa^{*}$ is either $\kappa$ or $\kappa^{\prime}$. Finally, $\mathcal{A}$ may make extraction queries, i.e., it is given oracle access to $\operatorname{Extract}\left(s k_{\varepsilon}, \cdot\right)$, subject to the constraint that it may not query any identity that is a prefix of (or equal to) the target identity $i d^{*}$. The advantage $\operatorname{Adv}_{\mathrm{HIBE}}^{\text {sid-ind-cpa }}(\mathcal{A})$ of $\mathcal{A}$ is its distinguishing advantage between the two cases $\kappa^{*}=\kappa$ and $\kappa^{*}=\kappa^{\prime}$.

Another notion is an adaptive-identity attack, in which the adversary is first given $m p k$ and oracle access to Extract $\left(s k_{\varepsilon}, \cdot\right)$ before choosing its target identity $i d^{*}$ (as before, under the constraint that no query identity be a prefix of $i d^{*}$ ). Finally, both notions may be extended to chosen-ciphertext attacks in the natural way; we omit precise definitions.

\subsection{Lattices}

In this work, we use $m$-dimensional full-rank integer lattices, which are discrete additive subgroups of $\mathbb{Z}^{m}$ having finite index, i.e., the quotient group $\mathbb{Z}^{m} / \Lambda$ is finite. A lattice $\Lambda \subseteq \mathbb{Z}^{m}$ can equivalently be defined as the set of all integer linear combinations of $m$ linearly independent basis vectors $\mathbf{B}=\left\{\mathbf{b}_{1}, \ldots, \mathbf{b}_{m}\right\} \subset \mathbb{Z}^{m}$ :

$$
\Lambda=\mathcal{L}(\mathbf{B})=\left\{\mathbf{B} \mathbf{c}=\sum_{i \in[m]} c_{i} \mathbf{b}_{i}: \mathbf{c} \in \mathbb{Z}^{m}\right\} .
$$

When $m \geq 2$, there are infinitely many bases that generate the same lattice.

Every lattice $\Lambda \subseteq \mathbb{Z}^{m}$ has a unique canonical basis $\mathbf{H}=\operatorname{HNF}(\Lambda) \in \mathbb{Z}^{m \times m}$ called its Hermite normal form (HNF). The only facts about the HNF that we require are that it is unique, and that it may be computed efficiently given an arbitrary basis $\mathbf{B}$ of the lattice (see [42] and references therein). We write $\operatorname{HNF}(\mathbf{B})$ to denote the Hermite normal form of the lattice generated by basis $\mathbf{B}$. 
The following lemma will be useful in our constructions.

Lemma 1 ([40, Lemma 7.1, page 129]). There is a deterministic poly-time algorithm ToBasis(S, B) that, given a full-rank set (not necessarily a basis) of lattice vectors $\mathbf{S} \subset \Lambda=\mathcal{L}(\mathbf{B})$, outputs a basis $\mathbf{T}$ of $\Lambda$ such that $\left\|\widetilde{\mathbf{t}_{i}}\right\| \leq\left\|\widetilde{\mathbf{s}_{i}}\right\|$ for all $i$.

Hard Lattices and Problems. We will work with an certain family of integer lattices whose importance in cryptography was first demonstrated by Ajtai [5]. Let $n \geq 1$ and modulus $q \geq 2$ be integers; the dimension $n$ is the main cryptographic security parameter throughout this work, and all other parameters are implicitly functions of $n$. An $m$-dimensional lattice from the family is specified relative to the additive group $\mathbb{Z}_{q}^{n}$ by a parity check (more accurately, "arity check") matrix $\mathbf{A} \in \mathbb{Z}_{q}^{n \times m}$. The associated lattice is defined as

$$
\Lambda^{\perp}(\mathbf{A})=\left\{\mathbf{x} \in \mathbb{Z}^{m}: \mathbf{A x}=\sum_{j \in[m]} x_{j} \cdot \mathbf{a}_{j}=\mathbf{0} \in \mathbb{Z}_{q}^{n}\right\} \subseteq \mathbb{Z}^{m} .
$$

One may check that $\Lambda^{\perp}(\mathbf{A})$ contains $q \mathbb{Z}^{m}$ (and in particular, the identity $\mathbf{0} \in \mathbb{Z}^{m}$ ) and is closed under addition, hence it is a full-rank subgroup of (and lattice in) $\mathbb{Z}^{m}$. For any $\mathbf{y}$ in the subgroup of $\mathbb{Z}_{q}^{n}$ generated by the columns of $\mathbf{A}$, we also define the coset

$$
\Lambda_{\mathbf{y}}^{\perp}(\mathbf{A})=\left\{\mathbf{x} \in \mathbb{Z}^{m}: \mathbf{A x}=\mathbf{y}\right\}=\Lambda^{\perp}(\mathbf{A})+\overline{\mathbf{x}},
$$

where $\overline{\mathbf{x}} \in \mathbb{Z}^{m}$ is an arbitrary element of $\Lambda_{\overline{\mathbf{x}}} \frac{}{\text {. }}$.

It is known (see, e.g., [51, Claim 5.3]) that for any fixed constant $C>1$ and any $m \geq C n \lg q$, the columns of a uniformly random $\mathbf{A} \in \mathbb{Z}_{q}^{n \times m}$ generate all of $\mathbb{Z}_{q}^{n}$, except with $2^{-\Omega(n)}=\operatorname{negl}(n)$ probability. (Moreover, the subgroup generated by $\mathbf{A}$ can be computed efficiently.) Therefore, throughout the paper we sometimes implicitly assume that such a uniform $\mathbf{A}$ generates $\mathbb{Z}_{q}^{n}$.

We recall the short integer solution (SIS) and learning with errors (LWE) problems, which may be seen as average-case problems related to the family of lattices described above.

Definition 1 (Short Integer Solution). An instance of the $\mathrm{SIS}_{q, \beta}$ problem (in the $\ell_{2}$ norm) is a uniformly random matrix $\mathbf{A} \in \mathbb{Z}_{q}^{n \times m}$ for any desired $m=\operatorname{poly}(n)$. The goal is to find a nonzero integer vector $\mathbf{v} \in \mathbb{Z}^{m}$ such that $\|\mathbf{v}\|_{2} \leq \beta$ and $\mathbf{A v}=\mathbf{0} \in \mathbb{Z}_{q}^{n}$, i.e., $\mathbf{v} \in \Lambda^{\perp}(\mathbf{A})$.

Let $\chi$ be some distribution over $\mathbb{Z}_{q}$. For a vector $\mathbf{v} \in \mathbb{Z}_{q}^{\ell}$ of any dimension $\ell \geq 1$, $\operatorname{Noisy}_{\chi}(\mathbf{v}) \in \mathbb{Z}_{q}^{\ell}$ denotes the vector obtained by adding (modulo $q$ ) independent samples drawn from $\chi$ to each entry of $\mathbf{v}$ (one sample per entry). For a vector $\mathbf{s} \in \mathbb{Z}_{q}^{n}, A_{\mathbf{s}, \chi}$ is the distribution over $\mathbb{Z}_{q}^{n} \times \mathbb{Z}_{q}$ obtained by choosing a vector $\mathbf{a} \in \mathbb{Z}_{q}^{n}$ uniformly at random and outputting (a, $\left.\operatorname{Noisy}_{\chi}(\langle\mathbf{a}, \mathbf{s}\rangle)\right)$. In this work (and most others relating to LWE), $\chi$ is always a discretized normal error distribution parameterized by some $\alpha \in(0,1)$, which is obtained by drawing $x \in \mathbb{R}$ from the Gaussian distribution of width $\alpha$ (i.e., $x$ is chosen with probability proportional to $\left.\exp \left(-\pi x^{2} / \alpha^{2}\right)\right)$ and outputting $\lfloor q \cdot x\rceil \bmod q$.

Definition 2 (Learning with Errors). The $\mathrm{LWE}_{q, \chi}$ problem is to distinguish, given oracle access to any desired $m=\operatorname{poly}(n)$ samples, between the distribution $A_{\mathbf{s}, \chi}$ (for uniformly random and secret $\mathbf{s} \in \mathbb{Z}_{q}^{n}$ ) and the uniform distribution over $\mathbb{Z}_{q}^{n} \times \mathbb{Z}_{q}$. 
We write $\operatorname{Adv}_{\mathrm{SIS}_{q, \beta}}(\mathcal{A})$ and $\operatorname{Adv}_{\mathrm{LWE}_{q, \chi}}(\mathcal{A})$ to denote the success probability and distinguishing advantage of an algorithm $\mathcal{A}$ for the SIS and LWE problems, respectively.

For appropriate parameters, solving SIS and LWE (on the average, with non-negligible advantage) is known to be as hard as approximating certain lattice problems, such as the (decision) shortest vector problem, in the worst case. Specifically, for $q \geq \beta$. $\omega(\sqrt{n \log n})$, solving $\operatorname{SIS}_{q, \beta}$ yields approximation factors of $\tilde{O}(\beta \cdot \sqrt{n})$ [41, 25]. For $q \geq(1 / \alpha) \cdot \omega(\sqrt{n \log n})$, solving $\operatorname{LWE}_{q, \chi}$ yields approximation factors of $\tilde{O}(n / \alpha)$ (in some cases, via a quantum reduction); see [51, 45] for precise statements.

Gaussians over Lattices. We briefly recall Gaussian distributions over lattices, specialized to the family described above; for more details see [41, 25]. For any $s>0$ and dimension $m \geq 1$, the Gaussian function $\rho_{s}: \mathbb{R}^{m} \rightarrow(0,1]$ is defined as $\rho_{s}(\mathbf{x})=$ $\exp \left(-\pi\|\mathbf{x}\|^{2} / s^{2}\right)$. For any coset $\Lambda_{\mathbf{y}}^{\perp}(\mathbf{A})$, the discrete Gaussian distribution $D_{\Lambda_{\mathbf{y}}(\mathbf{A}), s}$ (centered at zero) over the coset assigns probability proportional to $\rho_{s}(\mathbf{x})$ to each $\mathbf{x} \in \Lambda_{\mathbf{y}}^{\perp}(\mathbf{A})$, and probability zero elsewhere.

We summarize several standard facts from the literature about discrete Gaussians over lattices, again specialized to our family of interest.

Lemma 2. Let $\mathbf{S}$ be any basis of $\Lambda^{\perp}(\mathbf{A})$ for some $\mathbf{A} \in \mathbb{Z}_{q}^{n \times m}$ whose columns generate $\mathbb{Z}_{q}^{n}$, let $\mathbf{y} \in \mathbb{Z}_{q}^{n}$ be arbitrary, and let $s \geq\|\widetilde{\mathbf{S}}\| \cdot \omega(\sqrt{\log n})$.

1. [41. Lemma 4.4]: $\operatorname{Pr}_{\mathbf{x} \leftarrow D_{\Lambda \frac{1}{\mathbf{y}}(\mathbf{A}), s}}[\|\mathbf{x}\|>s \cdot \sqrt{m}] \leq \operatorname{negl}(n)$.

2. [47. Lemma 2.11]: $\operatorname{Pr}_{\mathbf{x} \leftarrow D_{\Lambda^{\perp}(\mathbf{A}), s}}[\mathbf{x}=\mathbf{0}] \leq \operatorname{negl}(n)$.

3. [51. Corollary 3.16]: a set of $O\left(m^{2}\right)$ independent samples from $D_{\Lambda^{\perp}(\mathbf{A}), s}$ contains a set of $m$ linearly independent vectors, except with $\operatorname{negl}(n)$ probability.

4. [25. Theorem 3.1]: For $\mathbf{x} \leftarrow D_{\mathbb{Z}^{m}, s}$, the marginal distribution of $\mathbf{y}=\mathbf{A x} \in \mathbb{Z}_{q}^{n}$ is uniform (up to $\operatorname{negl}(n)$ statistical distance), and the conditional distribution of $\mathbf{x}$ given $\mathbf{y}$ is $D_{\Lambda \perp(\mathbf{A}), s}$.

5. [25. Theorem 4.1]: there is a PPT algorithm $\operatorname{SampleD}(\mathbf{S}, \mathbf{y}, s)$ that generates a sample from $D_{\Lambda_{\mathbf{y}}^{\perp}(\mathbf{A}), s}$ (up to $\operatorname{negl}(n)$ statistical distance).

For Item 5] above, a recent work [46] gives an alternative SampleD algorithm that is more efficient and fully parallelizable; it works for any $s \geq \sigma_{1}(\mathbf{S}) \cdot \omega(\sqrt{\log n})$, where $\sigma_{1}(\mathbf{S})$ is the largest singular value of $\mathbf{S}$ (which is never less than $\|\widetilde{\mathbf{S}}\|$, but is also not much larger in most important cases; see [46] for details).

\section{Principles of Bonsai Trees}

In this section we lay out the framework and main techniques for the cultivation of bonsai trees. There are four basic principles: undirected growth, controlled growth, extending control over arbitrary new growth, and randomizing control.

\subsection{Undirected Growth}

Undirected growth is useful primarily for allowing a simulator to embed an underlying challenge problem (i.e., SIS or LWE) into a tree. This is done simply by drawing fresh 
uniformly random and independent samples $\mathbf{a}_{i} \in \mathbb{Z}_{q}^{n}$ from the problem distribution, and grouping them into (or appending them onto) a parity-check matrix $\mathbf{A}$.

More formally, let $\mathbf{A} \in \mathbb{Z}_{q}^{n \times m}$ be arbitrary for some $m \geq 0$, and let $\mathbf{A}^{\prime}=\mathbf{A} \| \overline{\mathbf{A}} \in$ $\mathbb{Z}_{q}^{n \times m^{\prime}}$ for some $m^{\prime}>m$ be an arbitrary extension of $\mathbf{A}$. Then it is easy to see that $\Lambda^{\perp}\left(\mathbf{A}^{\prime}\right) \subseteq \mathbb{Z}^{m^{\prime}}$ is a higher-dimensional superlattice of $\Lambda^{\perp}(\mathbf{A}) \subseteq \mathbb{Z}^{m}$, when the latter is lifted to $\mathbb{Z}^{m^{\prime}}$. Specifically, for any $\mathbf{v} \in \Lambda^{\perp}(\mathbf{A})$, the vector $\mathbf{v}^{\prime}=\mathbf{v} \| \mathbf{0} \in \mathbb{Z}^{m^{\prime}}$ is in $\Lambda^{\perp}\left(\mathbf{A}^{\prime}\right)$ because $\mathbf{A}^{\prime} \mathbf{v}^{\prime}=\mathbf{A v}=\mathbf{0} \in \mathbb{Z}_{q}^{n}$.

In fact, the columns of $\mathbf{A}^{\prime}$ may be ordered arbitrarily (e.g., the columns of $\overline{\mathbf{A}}$ may be both appended and prepended to $\mathbf{A}$ ), which simply results in the entries of the vectors in $\Lambda^{\perp}\left(\mathbf{A}^{\prime}\right)$ being permuted in the corresponding manner. That is, $\Lambda^{\perp}\left(\mathbf{A}^{\prime} \mathbf{P}\right)=\mathbf{P} \cdot \Lambda^{\perp}\left(\mathbf{A}^{\prime}\right)$ for any permutation matrix $\mathbf{P} \in\{0,1\}^{m^{\prime} \times m^{\prime}}$, because $\left(\mathbf{A}^{\prime} \mathbf{P}\right) \mathbf{x}=\mathbf{A}^{\prime}(\mathbf{P x}) \in \mathbb{Z}_{q}^{n}$ for all $\mathbf{x}=\mathbb{Z}^{m^{\prime}}$.

\subsection{Controlled Growth}

We say that an arborist controls a lattice if it knows a relatively good (i.e., short) basis for the lattice. The following lemma says that a random lattice from our family of interest can be generated under control 3

Proposition 1 ([6]). There is a fixed constant $C>1$ and a probabilistic polynomialtime algorithm GenBasis $\left(1^{n}, 1^{m}, q\right)$ that, for poly $(n)$-bounded $m \geq C n \lg q$, outputs $\mathbf{A} \in \mathbb{Z}_{q}^{n \times m}$ and $\mathbf{S} \in \mathbb{Z}^{m \times m}$ such that:

- the distribution of $\mathbf{A}$ is within $\operatorname{negl}(n)$ statistical distance of uniform,

- $\mathbf{S}$ is a basis of $\Lambda^{\perp}(\mathbf{A})$, and

- $\|\widetilde{\mathbf{S}}\| \leq \widetilde{L}=O(\sqrt{n \log q})$.

\subsection{Extending Control}

Here we describe how an arborist may extend its control of a lattice to an arbitrary higher-dimensional extension, without any loss of quality in the resulting basis.

Lemma 3. Let $\mathbf{S} \in \mathbb{Z}^{m \times m}$ be an arbitrary basis of $\Lambda^{\perp}(\mathbf{A})$ for some $\mathbf{A} \in \mathbb{Z}_{q}^{n \times m}$ whose columns generate the entire group $\mathbb{Z}_{q}^{n}$, and let $\overline{\mathbf{A}} \in \mathbb{Z}_{q}^{n \times \bar{m}}$ be arbitrary. There is a deterministic polynomial-time algorithm $\operatorname{ExtBasis}\left(\mathbf{S}, \mathbf{A}^{\prime}=\mathbf{A} \| \overline{\mathbf{A}}\right)$ that outputs a basis $\mathbf{S}^{\prime}$ of $\Lambda^{\perp}\left(\mathbf{A}^{\prime}\right) \subseteq \mathbb{Z}^{m+\bar{m}}$ such that $\left\|\widetilde{\mathbf{S}^{\prime}}\right\|=\|\widetilde{\mathbf{S}}\|$. Moreover, the statement holds even if the columns of $\mathbf{A}^{\prime}$ are permuted arbitrarily (e.g., if columns of $\overline{\mathbf{A}}$ are both appended and prepended to $\mathbf{A})$.

Proof. The ExtBasis $\left(\mathbf{S}, \mathbf{A}^{\prime}\right)$ algorithm computes and outputs an $\mathbf{S}^{\prime}$ of the form

$$
\mathbf{S}^{\prime}=\left(\begin{array}{cc}
\mathbf{S} & \mathbf{W} \\
\mathbf{0} & \mathbf{I}
\end{array}\right)
$$

\footnotetext{
${ }^{3}$ An earlier version of this paper [44] used an underlying lemma from [6] to directly extend a random parity-check matrix $\mathbf{A}$ (without known good basis) into a random $\mathbf{A}^{\prime}=\mathbf{A} \| \overline{\mathbf{A}}$ with known good basis. While that method saves a small constant factor in key sizes, the applications become somewhat more cumbersome to describe; moreover, our present approach is more general.
} 
where $\mathbf{I} \in \mathbb{Z}^{\bar{m} \times \bar{m}}$ is the identity matrix, and $\mathbf{W} \in \mathbb{Z}^{m \times \bar{m}}$ is an arbitrary (not necessarily short) solution to $\mathbf{A W}=-\overline{\mathbf{A}} \in \mathbb{Z}_{q}^{n \times \bar{m}}$. Note that $\mathbf{W}$ exists by hypothesis on $\mathbf{A}$, and may be computed efficiently using Gaussian elimination (for example).

We analyze $\mathbf{S}^{\prime}$. First, $\mathbf{A}^{\prime} \mathbf{S}^{\prime}=\mathbf{0}$ by assumption on $\mathbf{S}$ and by construction, so $\mathbf{S}^{\prime} \subset$ $\Lambda^{\perp}\left(\mathbf{A}^{\prime}\right)$. Moreover, $\mathbf{S}^{\prime}$ is a basis of $\Lambda^{\perp}\left(\mathbf{A}^{\prime}\right)$ : let $\mathbf{v}^{\prime}=\mathbf{v} \| \overline{\mathbf{v}} \in \Lambda^{\perp}\left(\mathbf{A}^{\prime}\right)$ be arbitrary, where $\mathbf{v} \in \mathbb{Z}^{m}, \overline{\mathbf{v}} \in \mathbb{Z}^{\bar{m}}$. Then we have

$$
\mathbf{0}=\mathbf{A}^{\prime} \mathbf{v}^{\prime}=\mathbf{A} \mathbf{v}+\overline{\mathbf{A}} \overline{\mathbf{v}}=\mathbf{A} \mathbf{v}-(\mathbf{A W}) \overline{\mathbf{v}}=\mathbf{A}(\mathbf{v}-\mathbf{W} \overline{\mathbf{v}}) \in \mathbb{Z}_{q}^{n} .
$$

Thus $\mathbf{v}-\mathbf{W} \overline{\mathbf{v}} \in \Lambda^{\perp}(\mathbf{A})$, so by assumption on $\mathbf{S}$ there exists some $\mathbf{z} \in \mathbb{Z}^{m}$ such that $\mathbf{S z}=\mathbf{v}-\mathbf{W} \overline{\mathbf{v}}$. Now let $\mathbf{z}^{\prime}=\mathbf{z} \| \overline{\mathbf{v}} \in \mathbb{Z}^{m+\bar{m}}$. By construction, we have

$$
\mathbf{S}^{\prime} \mathbf{z}^{\prime}=(\mathbf{S z}+\mathbf{W} \overline{\mathbf{v}})\|\overline{\mathbf{v}}=\mathbf{v}\| \overline{\mathbf{v}}=\mathbf{v}^{\prime} .
$$

Because $\mathbf{v}^{\prime} \in \Lambda^{\perp}\left(\mathbf{A}^{\prime}\right)$ was arbitrary, $\mathbf{S}^{\prime}$ is therefore a basis of $\Lambda^{\perp}\left(\mathbf{A}^{\prime}\right)$.

We next confirm that $\left\|\widetilde{\mathbf{S}^{\prime}}\right\|=\|\widetilde{\mathbf{S}}\|$. For every $i \in[m]$, we clearly have $\left\|\widetilde{\mathbf{s}_{i}^{\prime}}\right\|=$ $\left\|\widetilde{\mathbf{s}_{i}}\right\|$. Now because $\mathbf{S}$ is full-rank, we have $\operatorname{span}(\mathbf{S})=\operatorname{span}\left(\mathbf{e}_{1}, \ldots, \mathbf{e}_{m}\right) \subseteq \mathbb{R}^{m+\bar{m}}$. Therefore, for $i=m+1, \ldots, m+\bar{m}$ we have $\widetilde{\mathbf{s}_{i}^{\prime}}=\mathbf{e}_{i} \in \mathbb{R}^{m+\bar{m}}$, so $\left\|\widetilde{\mathbf{s}_{i}^{\prime}}\right\|=1 \leq\left\|\widetilde{\mathbf{s}_{1}^{\prime}}\right\|$, as desired.

For the final part of the lemma, we simply compute $\mathbf{S}^{\prime}$ for $\mathbf{A}^{\prime}=\mathbf{A} \| \overline{\mathbf{A}}$ as described above, and output $\mathbf{S}^{\prime \prime}=\mathbf{P} \mathbf{S}^{\prime}$ as a basis for $\Lambda^{\perp}\left(\mathbf{A}^{\prime} \mathbf{P}\right)$, where $\mathbf{P}$ is the desired permutation matrix. The Gram-Schmidt lengths remain unchanged, i.e., $\left\|\widetilde{\mathbf{s}_{i}^{\prime \prime}}\right\|=\left\|\widetilde{\mathbf{s}_{i}^{\prime}}\right\|$, because $\mathbf{P}$ is orthogonal and hence the right-triangular matrices are exactly the same in the QR decompositions of $\mathbf{S}^{\prime}$ and $\mathbf{P} \mathbf{S}^{\prime}$.

An Optimization. In many of our cryptographic applications, a common design pattern is to extend a basis $\mathbf{S}$ of an $m$-dimensional lattice $\Lambda^{\perp}(\mathbf{A})$ to a basis $\mathbf{S}^{\prime}$ of a dimension$m^{\prime}$ superlattice $\Lambda^{\perp}\left(\mathbf{A}^{\prime}\right)$, and then immediately sample (one or more times) from a discrete Gaussian over the superlattice. For the construction and analysis of our schemes, it is more convenient and modular to treat these operations separately; however, a naive implementation would be rather inefficient, requiring at least $\left(m^{\prime}\right)^{2}$ space and time (where $m^{\prime}$ can be substantially larger than $m$ ). Fortunately, the special structure of the extended basis $\mathbf{S}^{\prime}$, together with the recursive "nearest-plane" operation of the SampleD algorithm from [25], can be exploited to avoid any explicit computation of $\mathbf{S}^{\prime}$, thus saving a significant amount of time and space over the naive approach.

Let $\mathbf{S} \in \mathbb{Z}^{m \times m}$ be a basis of $\Lambda^{\perp}(\mathbf{A})$, and let $\mathbf{A}^{\prime}=\mathbf{A} \| \overline{\mathbf{A}}$ for some $\overline{\mathbf{A}} \in \mathbb{Z}_{q}^{n \times \bar{m}}$, where $m^{\prime}=m+\bar{m}$. Consider a hypothetical execution of $\operatorname{SampleD}\left(\mathbf{S}^{\prime}, \mathbf{y}^{\prime}, s\right)$, where $\mathbf{S}^{\prime}=\left(\begin{array}{cc}\mathbf{S} & \mathbf{W} \\ \mathbf{0} & \mathbf{I}\end{array}\right)$ is the extended basis as described in the proof of Lemma 3 . Recall that for all $i=m+1, \ldots, m^{\prime}$, the vectors $\mathbf{s}_{i}^{\prime}$ are integral and have unit Gram-Schmidt vectors $\widetilde{\mathbf{s}_{i}^{\prime}}=\mathbf{e}_{i}$. By inspection, it can be verified that a recursive execution of $\operatorname{SampleD}\left(\mathbf{S}^{\prime}, \mathbf{y}^{\prime}, s\right)$ simply ends up choosing all the entries of $\overline{\mathbf{v}} \in \mathbb{Z}^{\bar{m}}$ independently from $D_{\mathbb{Z}, s}$, then choosing $\mathbf{v} \leftarrow \operatorname{SampleD}\left(\mathbf{S}, \mathbf{y}^{\prime}-\overline{\mathbf{A}} \overline{\mathbf{v}}, s\right)$, and outputting $\mathbf{v}^{\prime}=\mathbf{v} \| \overline{\mathbf{v}}$. Therefore, the optimized algorithm can perform exactly the same steps, thus avoiding any need to compute and store $\mathbf{W}$ itself. A similar optimization also works for any permutation of the columns of $\mathbf{A}^{\prime}$. 
In the language of the "preimage sampleable" function $f_{\mathbf{A}}(\mathbf{v})=\mathbf{A} \mathbf{v} \in \mathbb{Z}_{q}^{n}$ defined in [25], the process described above corresponds to sampling a preimage from $f_{\mathbf{A}^{\prime}}^{-1}\left(\mathbf{y}^{\prime}\right)$ by first computing $\overline{\mathbf{y}}=f_{\overline{\mathbf{A}}}(\overline{\mathbf{v}})=\overline{\mathbf{A}} \overline{\mathbf{v}} \in \mathbb{Z}_{q}^{n}$ in the "forward" direction (for random $\left.\overline{\mathbf{v}} \leftarrow \mathbb{D}_{\mathbb{Z}^{\bar{m}}, s}\right)$, then choosing a random preimage $\mathbf{v} \leftarrow f_{\mathbf{A}}^{-1}\left(\mathbf{y}^{\prime}-\overline{\mathbf{y}}\right)$ under the appropriate distribution, and outputting $\mathbf{v}^{\prime}=\mathbf{v} \| \overline{\mathbf{v}}$

\subsection{Randomizing Control}

Finally, we show how an arborist can randomize its lattice basis, with a slight loss in quality. This operation is useful for securely delegating control to another entity, because the resulting basis is still short, but is statistically independent (essentially) of the original basis.

The probabilistic polynomial-time algorithm $\operatorname{RandBasis}(\mathbf{S}, s)$ takes a basis $\mathbf{S}$ of an $m$-dimensional integer lattice $\Lambda$ and a parameter $s \geq\|\widetilde{\mathbf{S}}\| \cdot \omega(\sqrt{\log n})$, and outputs a basis $\mathbf{S}^{\prime}$ of $\Lambda$, generated as follows.

1. Let $i \leftarrow 0$. While $i<m$,

(a) Choose $\mathbf{v} \leftarrow$ SampleD $(\mathbf{S}, s)$. If $\mathbf{v}$ is linearly independent of $\left\{\mathbf{v}_{1}, \ldots, \mathbf{v}_{i}\right\}$, then let $i \leftarrow i+1$ and let $\mathbf{v}_{i}=\mathbf{v}$.

2. Output $\mathbf{S}^{\prime}=\operatorname{ToBasis}(\mathbf{V}, \operatorname{HNF}(\mathbf{S}))$.

In the final step, the (unique) Hermite normal form basis $\operatorname{HNF}(\mathbf{S})$ of $\Lambda$ is used to ensure that no information particular to $\mathbf{S}$ is leaked by the output; any other publicly available (or publicly computable) basis of the lattice could also be used in its place.

Lemma 4. With overwhelming probability, $\mathbf{S}^{\prime} \leftarrow$ RandBasis $(\mathbf{S}, s)$ repeats Step 1 a at most $O\left(m^{2}\right)$ times, and $\left\|\mathbf{S}^{\prime}\right\| \leq s \cdot \sqrt{m}$. Moreover, for any two bases $\mathbf{S}_{0}, \mathbf{S}_{1}$ of the same lattice and any $s \geq \max \left\{\left\|\widetilde{\mathbf{S}_{0}}\right\|,\left\|\widetilde{\mathbf{S}_{1}}\right\|\right\} \cdot \omega(\sqrt{\log n})$, the outputs of $\operatorname{RandBasis}\left(\mathbf{S}_{0}, s\right)$ and $\operatorname{RandBasis}\left(\mathbf{S}_{1}, s\right)$ are within $\operatorname{negl}(n)$ statistical distance.

Proof. The bound on $\left\|\mathbf{S}^{\prime}\right\|$ and on the number of iterations follow immediately from Lemma 2 items 1 and 3 respectively. The claim on the statistical distance follows from the fact that each sample $\mathbf{v}$ drawn in Step 1a has the same distribution (up to negl $(n)$ statistical distance) whether $\mathbf{S}_{0}$ or $\mathbf{S}_{1}$ is used, and the fact that $\operatorname{HNF}\left(\mathbf{S}_{0}\right)=\operatorname{HNF}\left(\mathbf{S}_{1}\right)$ because the Hermite normal form of a lattice is unique.

\section{Signatures}

Here we use bonsai tree principles to construct a signature scheme that is existentially unforgeable under a static chosen-message attack (i.e., eu-scma-secure). As discussed in Section 2.2, this suffices (using chameleon hashing) for the construction of an (offline / online) signature scheme that is unforgeable under adaptive chosen-message attack (eu-acma-secure).

\footnotetext{
${ }^{4}$ An earlier version of this paper [17] explicitly defined a sampling procedure using this perspective, and gave a (somewhat involved) proof that it correctly samples from a discrete Gaussian over $\Lambda^{\perp}\left(\mathbf{A}^{\prime}\right)$. Here, correctness follows directly by examining the operation of SampleD on the structured basis $\mathbf{S}^{\prime}$.
} 
Our scheme involves a few parameters:

- a dimension $m=O(n \lg q)$ and a bound $\widetilde{L}=O(\sqrt{n \lg q})$, as per Proposition 1

- a (hashed) message length $k$, which induces a 'total dimension' $m^{\prime}=m \cdot(k+1)$;

- a Gaussian parameter $s=\widetilde{L} \cdot \omega(\sqrt{\log n})$.

The scheme SIG is defined as follows.

- Gen: using Proposition 1 generate $\mathbf{A}_{0} \in \mathbb{Z}_{q}^{n \times m}$ that is (negligibly close to) uniform, together with a basis $\mathbf{S}_{0}$ of $\Lambda^{\perp}\left(\mathbf{A}_{0}\right)$ such that $\left\|\widetilde{\mathbf{S}_{0}}\right\| \leq \widetilde{L}$. (Recall that the columns of $\mathbf{A}_{0}$ generate all of $\mathbb{Z}_{q}^{n}$, with overwhelming probability.)

Then for each $(b, j) \in\{0,1\} \times[k]$, choose uniformly random and independent $\mathbf{A}_{j}^{(b)} \in \mathbb{Z}_{q}^{n \times m}$. Output $v k=\left(\mathbf{A}_{0},\left\{\mathbf{A}_{j}^{(b)}\right\}\right)$ and $s k=\left(\mathbf{S}_{0}, v k\right)$.

- $\operatorname{Sign}\left(s k, \mu \in\{0,1\}^{k}\right)$ : let $\mathbf{A}_{\mu}=\mathbf{A}_{0}\left\|\mathbf{A}_{1}^{\left(\mu_{1}\right)}\right\| \cdots \| \mathbf{A}_{k}^{\left(\mu_{k}\right)} \in \mathbb{Z}_{q}^{n \times m^{\prime}}$. Output $\mathbf{v} \leftarrow$ $D_{\Lambda^{\perp}\left(\mathbf{A}_{\mu}\right), s}$, via

$$
\mathbf{v} \leftarrow \text { SampleD }\left(\operatorname{ExtBasis}\left(\mathbf{S}_{0}, \mathbf{A}_{\mu}\right), \mathbf{0}, s\right) .
$$

(In the rare event that $\mathbf{v}=\mathbf{0}$ or $\|\mathbf{v}\|>s \cdot \sqrt{m^{\prime}}$ (Lemma2], items 2] and 2), resample v. Note also that the optimization of Section 3.3 applies here.)

- $\operatorname{Ver}(v k, \mu, \mathbf{v})$ : let $\mathbf{A}_{\mu}$ be as above. Accept if $\mathbf{v} \neq \mathbf{0},\|\mathbf{v}\| \leq s \cdot \sqrt{m^{\prime}}$, and $\mathbf{v} \in$ $\Lambda^{\perp}\left(\mathbf{A}_{\mu}\right)$; else, reject.

Completeness is by inspection. Note that the matrix $\mathbf{A}_{0}$ can be omitted from the above scheme (thus making the total dimension $m \cdot k$ ), at the expense of a secret key that contains two short bases $\mathbf{S}_{1}^{(b)}$ of $\Lambda^{\perp}\left(\mathbf{A}_{1}^{(b)}\right)$, for $b=0,1$. The scheme's algorithms and security proof are easy to modify accordingly.

\subsection{Security}

Theorem 1. There exists a PPT oracle algorithm (a reduction) $\mathcal{S}$ attacking the $\mathrm{SIS}_{q, \beta}$ problem for $\beta=s \cdot \sqrt{m^{\prime}}$ such that, for any adversary $\mathcal{F}$ mounting an eu-scma attack on SIG and making at most $Q$ queries,

$$
\operatorname{Adv}_{\mathrm{SIS}_{q, \beta}}\left(\mathcal{S}^{\mathcal{F}}\right) \geq \mathbf{A d v}_{S I G}^{e u-s c m a}(\mathcal{F}) /(k \cdot Q)-\operatorname{negl}(n) .
$$

Proof. Let $\mathcal{F}$ be an adversary mounting an eu-scma attack on SIG. We construct a reduction $\mathcal{S}$ attacking $\mathrm{SIS}_{q, \beta}$. The reduction $\mathcal{S}$ takes as input $m^{\prime \prime}=m \cdot(2 k+1)$ uniformly random and independent samples from $\mathbb{Z}_{q}^{n}$ in the form of a matrix $\mathbf{A} \in$ $\mathbb{Z}_{q}^{n \times m^{\prime \prime}}$, parsing $\mathbf{A}$ as

$$
\mathbf{A}=\mathbf{A}_{0}\left\|\mathbf{U}_{1}^{(0)}\right\| \mathbf{U}_{1}^{(1)}\|\cdots\| \mathbf{U}_{k}^{(0)} \| \mathbf{U}_{k}^{(1)}
$$

for matrices $\mathbf{A}_{0}, \mathbf{U}_{i}^{(b)} \in \mathbb{Z}_{q}^{n \times m}$.

$\mathcal{S}$ simulates the static chosen-message attack to $\mathcal{F}$ as follows. First, $\mathcal{S}$ invokes $\mathcal{F}$ to receive $Q$ messages $\mu^{(1)}, \ldots, \mu^{(Q)} \in\{0,1\}^{k}$. (We may assume without loss of generality that $\mathcal{F}$ makes exactly $Q$ queries.) Then $\mathcal{S}$ computes the set $P$ of all strings $p \in\{0,1\}^{\leq k}$ having the property that $p$ is a shortest string for which no $\mu^{(j)}$ has $p$ as a prefix. In brief, each $p$ corresponds to a maximal subtree of $\{0,1\} \leq k$ (viewed as a tree) 
that does not contain any of the queried messages. The set $P$ may be computed efficiently via a breadth-first pruned search of $\{0,1\} \leq k$. Namely, starting from a queue initialized to $\{\varepsilon\}$, repeat the following until the queue is empty: remove the next string $p$ from the queue and test whether it is the prefix of any $\mu^{(j)}$; if not, add $p$ to $P$, else if $|p|<k$, add $p\|0, p\| 1 \in\{0,1\}^{\leq k}$ to the queue. Note that this algorithm runs in polynomial time because the only strings ever placed in the queue are prefixes of $\mu^{(j)}$, and hence there are at most $k \cdot Q$ strings in the set.

Next, $\mathcal{S}$ chooses some $p$ from $P$ uniformly at random, letting $t=|p|$. It then provides an SIG verification key $v k=\left(\mathbf{A}_{0},\left\{\mathbf{A}_{j}^{(b)}\right\}\right)$ to $\mathcal{F}$, generated as follows:

- Uncontrolled growth: for each $i \in[t]$, let $\mathbf{A}_{i}^{\left(p_{i}\right)}=\mathbf{U}_{i}^{(0)}$. For $i=t+1, \ldots, k$, and $b \in\{0,1\}$, let $\mathbf{A}_{i}^{(b)}=\mathbf{U}_{i}^{(b)}$.

- Controlled growth: for each $i \in[t]$, invoke Proposition 1 to generate $\mathbf{A}_{i}^{\left(1-p_{i}\right)}$ and basis $\mathbf{S}_{i}$ of $\Lambda^{\perp}\left(\mathbf{A}_{i}^{1-p_{i}}\right)$ such that $\left\|\widetilde{\mathbf{S}_{i}}\right\| \leq \widetilde{L}$.

Next, $\mathcal{S}$ generates signatures for each queried message $\mu=\mu^{(j)}$ as follows: let $i \in[t]$ be the first position at which $\mu_{i} \neq p_{i}$ (such $i$ exists by construction of $p$ ). Then $\mathcal{S}$ generates the signature $\mathbf{v} \leftarrow D_{\Lambda^{\perp}\left(\mathbf{A}_{\mu}\right), s}$ as

$$
\mathbf{v} \leftarrow \text { SampleD }\left(\operatorname{ExtBasis}\left(\mathbf{S}_{i}, \mathbf{A}_{\mu}\right), \mathbf{0}, s\right),
$$

where $\mathbf{A}_{\mu}=\mathbf{A}_{L}\left\|\mathbf{A}_{i}^{\left(1-p_{i}\right)}\right\| \mathbf{A}_{R}$ (for some matrices $\mathbf{A}_{L}, \mathbf{A}_{R}$ ) is as in the signature scheme, and has the form required by ExtBasis. (In the event that $\mathbf{v}=\mathbf{0}$ or $\|\mathbf{v}\|>\beta=$ $s \cdot \sqrt{m^{\prime}}$, resample v.)

Finally, if $\mathcal{F}$ produces a valid forgery $\left(\mu^{*}, \mathbf{v}^{*} \neq \mathbf{0}\right)$, then we have $\mathbf{v}^{*} \in \Lambda^{\perp}\left(\mathbf{A}_{\mu^{*}}\right)$, for $\mathbf{A}_{\mu^{*}}$ as defined in the scheme. First, $\mathcal{S}$ checks whether $p$ is a prefix of $\mu^{*}$. If not, $\mathcal{S}$ aborts; otherwise, note that $\mathbf{A}_{\mu^{*}}$ is the concatenation of $\mathbf{A}_{0}$ and $k$ blocks $\mathbf{U}_{i}^{(b)}$. Therefore, by inserting zeros into $\mathbf{v}^{*}, \mathcal{S}$ can generate a nonzero $\mathbf{v} \in \mathbb{Z}^{m^{\prime \prime}}$ so that $\mathbf{A v}=\mathbf{0} \in \mathbb{Z}_{q}^{n}$. Finally, $\mathcal{S}$ outputs $\mathbf{v}$ as a solution to SIS.

We now analyze the reduction. First observe that conditioned on any choice of $p \in P$, the verification key $v k$ given to $\mathcal{F}$ is negligibly close to uniform, and the signatures given to $\mathcal{F}$ are distributed exactly as in the real attack (up to negligible statistical distance), by Lemma 2 and the fact that $s \geq\left\|\widetilde{\mathbf{S}_{i}}\right\| \cdot \omega(\sqrt{\log n})$. Therefore, $\mathcal{F}$ outputs a valid forgery $\left(\mu^{*}, \mathbf{v}^{*} \neq \mathbf{0}\right)$ with probability at least $\mathbf{A d v}_{\text {SIG }}^{\text {eu-scma }}(\mathcal{F})-\operatorname{negl}(n)$. Finally, conditioned on the forgery, the choice of $p \in P$ is still negligibly close to uniform, so $p$ is a prefix of $\mu^{*}$ with probability at least $1 /(k \cdot Q)-\operatorname{negl}(n)$. In such a case, $\mathbf{A v}=\mathbf{0}$ and $\|\mathbf{v}\|=\left\|\mathbf{v}^{*}\right\| \leq \beta$ by construction, hence $\mathbf{v}$ is a valid solution to the given SIS instance, as desired.

\section{Hierarchical ID-Based Encryption}

\subsection{Key Encapsulation Mechanism}

For our HIBE schemes, it is convenient and more modular to abstract away the encryption and decryption processes into a key-encapsulation mechanism (KEM). The 
following LWE-based KEM from [25] (which is dual to the scheme of Regev [51]) is now standard. The reader need not be concerned with the details in order to progress to the HIBE schemes; it is enough simply to understand the KEM interface (i.e., the public/secret keys and ciphertext).

KEM is parametrized by a modulus $q$, dimension $m$, key length $\ell$, and Gaussian parameter $s$ that determines the error distribution $\chi$ used for encapsulation. As usual, all these parameters are functions of the LWE dimension $n$, and are instantiated based on the particular context in which the KEM is used.

- KEM.Gen: Choose $\mathbf{A} \leftarrow \mathbb{Z}_{q}^{n \times m}$ uniformly at random, e $\leftarrow D_{\mathbb{Z}^{m}, s}$ and set $\mathbf{y}=$ Ae $\in \mathbb{Z}_{q}^{n}$. Output public key $p k=(\mathbf{A}, \mathbf{y}) \in \mathbb{Z}_{q}^{n \times(m+1)}$ and secret key $s k=\mathbf{e}$.

- KEM.Encaps $(p k=(\mathbf{A}, \mathbf{y}))$ : Choose $\mathbf{s} \leftarrow \mathbb{Z}_{q}^{n}$ and let

$$
\mathbf{b} \leftarrow \operatorname{Noisy}_{\chi}\left(\mathbf{A}^{t} \mathbf{s}\right) \quad \text { and } \quad p \leftarrow \operatorname{Noisy}_{\chi}\left(\mathbf{y}^{t} \mathbf{s}+k \cdot\lfloor q / 2\rfloor\right),
$$

where $k \in\{0,1\}$ is a random bit. Output the key bit $k$ and ciphertext $(\mathbf{b}, p) \in$ $\mathbb{Z}_{q}^{m+1}$.

- KEM.Decaps $(s k=\mathbf{e},(\mathbf{b}, p))$ : Compute $p-\mathbf{e}^{t} \mathbf{b} \bmod q$ and output 0 if the result is closer to 0 than $\lfloor q / 2\rfloor$ modulo $q$, and 1 otherwise.

As explained in [25], the basic scheme can be amortized to allow for KEM keys of length $\ell=\operatorname{poly}(n)$ bits, with ciphertexts in $\mathbb{Z}_{q}^{m+\ell}$ and public keys in $\mathbb{Z}_{q}^{n \times(m+\ell)}$. This is done by including $\ell$ syndromes $\mathbf{y}_{1}, \ldots, \mathbf{y}_{\ell}$ (where $\mathbf{y}_{i}=\mathbf{A e}_{i}$ for independent $\mathbf{e}_{i} \leftarrow$ $\left.D_{\mathbb{Z}^{m}, s}\right)$ in the public key, and concealing one KEM bit with each of them using the same $\mathbf{s}$ and $\mathbf{b} \leftarrow$ Noisy $_{\chi}\left(\mathbf{A}^{t} \mathbf{s}\right)$. Furthermore, it is also possible to conceal $\Omega(\log n)$ KEM bits per syndrome, which yields an amortized expansion factor of $O(1)$. For simplicity, in this work we deal only with the case of single-bit encapsulation, but all of our schemes can be amortized in a manner similar to the above.

We point out one nice property of KEM, which is convenient for the security proof of our BTE/HIBE schemes: for any dimensions $m \leq m^{\prime}$ (and leaving all other parameters the same), the adversary's view for dimension $m$ may be produced by taking a view for dimension $m^{\prime}$, and truncating the values $\mathbf{A} \in \mathbb{Z}_{q}^{n \times m^{\prime}}$ and $\mathbf{b} \in \mathbb{Z}_{q}^{m^{\prime}}$ to their first $m$ (out of $m^{\prime}$ ) components.

The following lemma is standard from prior work.

Lemma 5 (Correctness and Security). Let $m \geq C n \lg q$ for any fixed constant $C>1$, let $q \geq 4 s(m+1)$, and let $\chi$ be the discretized Gaussian of parameter $\alpha$ for $1 / \alpha \geq$ $s \sqrt{m+1} \cdot \omega(\sqrt{\log n})$. Then KEM.Decaps is correct with overwhelming probability over all the randomness of KEM.Gen and KEM.Encaps. Moreover, there exists a PPT oracle algorithm (a reduction) $\mathcal{S}$ attacking the $\mathrm{LWE}_{q, \chi}$ problem such that, for any adversary $\mathcal{A}$ mounting an ind-cpa attack on KEM,

$$
\operatorname{Adv}_{\operatorname{LWE}_{q, \chi}}\left(\mathcal{S}^{\mathcal{A}}\right) \geq \operatorname{Adv}_{K E M}^{\text {ind-cpa }}(\mathcal{A})-\operatorname{negl}(n) .
$$

\subsection{BTE and HIBE Scheme}

Our main construction in this section is a binary tree encryption (BTE) scheme, which suffices for full HIBE by hashing the components of the identities with a universal 
one-way or collision-resistant hash function [16]. We mainly focus on the case of selective-identity, chosen-plaintext attacks, i.e., sid-ind-cpa security.

The BTE scheme is parametrized by a dimension $m=O(n \lg q)$ as per Proposition 1 as well as a few quantities that are indexed by depth within the hierarchy. For an identity at depth $i \geq 0$ (where $i=0$ corresponds to the root),

$-(i+1) m$ is the dimension of a lattice associated with the identity;

- $\widetilde{L_{i}}$ is an upper bound on the Gram-Schmidt lengths of its secret short basis;

- for $i \geq 1, s_{i}$ is the Gaussian parameter used to generate that secret basis, which must exceed $\widetilde{L_{j}} \cdot \omega(\sqrt{\log n})$ for all $j<i$.

These parameters, along with the total depth $d$ of the hierarchy (or more accurately, the maximum number of delegations down any chain of authority), determine the modulus $q$ and error distribution $\chi$ used in the cryptosystem. We instantiate all the parameters after describing the scheme.

- BTE.Setup $(d)$ : Generate (via Proposition 1) $\mathbf{A}_{0} \in \mathbb{Z}_{q}^{n \times m}$ that is (negligibly close to) uniform with a basis $\mathbf{S}_{0}$ of $\Lambda^{\perp}\left(\mathbf{A}_{0}\right)$ such that $\|\widetilde{\mathbf{S}}\| \leq \widetilde{L_{0}}$. For each $(b, j) \in$ $\{0,1\} \times[d]$, generate uniform and independent $\mathbf{A}_{j}^{(b)} \in \mathbb{Z}_{q}^{n \times m}$. Choose $\mathbf{y} \in \mathbb{Z}_{q}^{n}$ uniformly at random. Output $m p k=\left(\mathbf{A}_{0},\left\{\mathbf{A}_{j}^{(b)}\right\}, \mathbf{y}, d\right)$ and $m s k=\mathbf{S}_{0}$.

All remaining algorithms implicitly take the master public key $m p k$ as input. For an identity $i d=\left(i d_{1}, \ldots, i d_{t}\right)$ of length $t=|i d| \leq d$, let

$$
\mathbf{A}_{i d}=\mathbf{A}_{0}\left\|\mathbf{A}_{1}^{\left(i d_{1}\right)}\right\| \cdots \| \mathbf{A}_{t}^{\left(i d_{t}\right)} \in \mathbb{Z}_{q}^{n \times(t+1) m},
$$

and let $p k_{i d}=\left(\mathbf{A}_{i d}, \mathbf{y}\right)$ denote the KEM public key associated with identity $i d$.

- BTE.Extract $\left(s k_{i d}=\left(\mathbf{S}_{i d}, \mathbf{e}_{i d}\right), i d^{\prime}=i d \| \overline{i d}\right)$ : if $t^{\prime}=\left|i d^{\prime}\right|>d$, output $\perp$. Else, let $t=|i d|$ and $\bar{t}=|\overline{i d}|$, and choose

$$
\left.\mathbf{S}_{i d^{\prime}} \leftarrow \text { RandBasis(ExtBasis }\left(\mathbf{S}_{i d}, \mathbf{A}_{i d^{\prime}}\right), s_{t^{\prime}}\right) .
$$

(Note that $s_{t^{\prime}} \geq \widetilde{L_{t}} \cdot \omega(\sqrt{\log n}) \geq\left\|\widetilde{\mathbf{S}_{i d}}\right\| \cdot \omega(\sqrt{\log n})$, as required by RandBasis.) Sample $\mathbf{e}_{i d^{\prime}} \leftarrow D_{\Lambda_{\mathbf{y}}\left(\mathbf{A}_{i d^{\prime}}\right), s_{t^{\prime}}}$ using SampleD(ExtBasis $\left.\left(\mathbf{S}_{i d}, \mathbf{A}_{i d^{\prime}}\right), \mathbf{y}_{i d^{\prime}}, s_{t^{\prime}}\right)$ and output $s k_{i d^{\prime}}=\left(\mathbf{S}_{i d^{\prime}}, \mathbf{e}_{i d^{\prime}}\right)$.

- BTE.Encaps $(i d)$ : Output $(k, C) \leftarrow \operatorname{KEM} . \operatorname{Encaps}\left(p k_{i d}\right)$.

- BTE.Decaps $\left(s k_{i d}=\left(\mathbf{S}_{i d}, \mathbf{e}_{i d}\right), C\right)$ : Output $k \leftarrow \operatorname{KEM} . \operatorname{Decaps}\left(\mathbf{e}_{i d}, C\right)$.

A multi-bit BTE follows in the same way from the multi-bit KEM scheme by using multiple uniform syndromes $\mathbf{y}_{i} \in \mathbb{Z}_{q}^{n}$, one for each bit of the KEM key.

Instantiating the parameters. Suppose that BTE is employed in a setting in which BTE.Extract $\left(s k_{i d}, i d^{\prime}\right)$ is invoked only on identities $i d^{\prime}$ whose lengths are a multiple of some $k \geq 1$. For example, consider the two main applications of [16]: in the forward-secure encryption scheme we have $k=1$, while in the generic BTE-to-HIBE transformation, $k$ is the output length of some UOWHF.

It is enough to define $s_{i}$ and $\widetilde{L_{i}}$ for $i$ that are multiples of $k$. Let

$$
\widetilde{L_{i}}=s_{i} \cdot \sqrt{(i+1) m}=s_{i} \cdot O(\sqrt{d \cdot n \lg q})
$$


be the bound on the Gram-Schmidt lengths of the secret bases (and note that this bound is satisfied with overwhelming probability by Lemma2 2 . Define $s_{i}=\widetilde{L_{i-k}} \cdot \omega(\sqrt{\log n})$, and unwind the recurrence to obtain

$$
\widetilde{L_{t}}=\widetilde{L_{0}} \cdot O(\sqrt{d \cdot n \lg q})^{t / k} \cdot \omega(\sqrt{\log n})^{t / k},
$$

with $\widetilde{L_{0}}=O(\sqrt{n \lg q})$ by Proposition 1

Finally, to ensure that the underlying KEM is complete (Lemma 5), we let $q \geq$ $4 s_{d} \cdot(d+2) m$ and $1 / \alpha=s_{d} \cdot \sqrt{(d+2) m} \cdot \omega(\sqrt{\log n})$. (It is also possible to use a different noise parameter for each level of the hierarchy.) For any $d=\operatorname{poly}(n)$, invoking known worst-case to average-case reductions for LWE yields an underlying approximation factor of $\tilde{O}(n / \alpha)=n \cdot \tilde{O}((d / k) \cdot \sqrt{n k}))^{d / k}$ for worst-case lattice problems.

Extensions: Anonymity and chosen-ciphertext security. With a small modification, BTE may be made anonymous across all depths of the hierarchy. That is, a ciphertext hides (computationally) the particular identity to which it was encrypted. The modification is simply to extend the $\mathbf{b}$ component of the KEM ciphertext to have length exactly $(d+1) m$, by padding it with enough uniformly random and independent elements of $\mathbb{Z}_{q}$. (The decryption algorithm simply ignores the padding.) Anonymity then follows immediately by the pseudorandomness of the LWE distribution.

Security under chosen-ciphertext attack (sid-ind-cca or aid-ind-cca) follows directly by a transformation of [10], from ind-cpa-secure HIBE for depth $d+1$ to ind-cca-secure HIBE for depth $d$.

Theorem 2 (Security of BTE). There exists a PPT oracle algorithm (a reduction) $\mathcal{S}$ attacking KEM (instantiated with dimension $(d+1) m$ and $q, \chi$ as in BTE) such that, for any adversary $\mathcal{A}$ mounting an atk attack on BTE,

$$
\operatorname{Adv}_{K E M}\left(\mathcal{S}^{\mathcal{A}}\right) \geq \operatorname{Adv}_{B T E}^{\text {sid-ind-cpa }}(\mathcal{A})-\operatorname{negl}(n) .
$$

Proof. Let $\mathcal{A}$ be an adversary mounting a sid-ind-cpa-attack on BTE. We construct a reduction $\mathcal{S}$ attacking KEM. It is given a uniformly random public key $p k=(\mathbf{A}, \mathbf{y}) \in$ $\mathbb{Z}_{q}^{n \times(d+1) m} \times \mathbb{Z}_{q}^{n}$, an encapsulation $(\mathbf{b}, p) \in \mathbb{Z}_{q}^{(d+1) m} \times \mathbb{Z}_{q}$, and a bit $k$ which either is encapsulated by $(\mathbf{b}, p)$ or is uniform and independent; the goal of $\mathcal{S}$ is to determine which is the case.

$\mathcal{S}$ simulates the (selective-identity) attack on BTE to $\mathcal{A}$ as follows. First, $\mathcal{S}$ invokes $\mathcal{A}$ on $1^{d}$ to receive its challenge identity $i d^{*}$ of length $t^{*}=\left|i d^{*}\right| \in[d]$. Then $\mathcal{S}$ produces a master public key $m p k$, encapsulated key, and some secret internal state as follows:

- Parsing the KEM inputs. Parse $\mathbf{A}$ as $\mathbf{A}=\mathbf{A}_{0}\left\|\mathbf{A}_{1}\right\| \cdots \| \mathbf{A}_{d} \in \mathbb{Z}_{q}^{n \times(d+1) m}$ for $\mathbf{A}_{i} \in \mathbb{Z}_{q}^{n \times m}$ for all $i \in\{0, \ldots, d\}$. Similarly, truncate $\mathbf{b}$ to $\mathbf{b}^{*} \in \mathbb{Z}_{q}^{\left(t^{*}+1\right) m}$.

- Undirected growth. For each $i \in\left[t^{*}\right]$, let $\mathbf{A}_{i}^{\left(i d_{i}^{*}\right)}=\mathbf{A}_{i}$.

- Controlled growth. For each $i \in\left[t^{*}\right]$, generate $\mathbf{A}_{i}^{\left(1-i d_{i}^{*}\right)} \in \mathbb{Z}_{q}^{n \times m}$ and basis $\mathbf{S}_{i}$ by invoking GenBasis $\left(1^{n}, 1^{m}, q\right)$. If $t^{*}<d$, for each $b \in\{0,1\}$ generate $\mathbf{A}_{t^{*}+1}^{(b)}$ and basis $\mathbf{S}_{t^{*}+1}^{(b)}$ by two independent invocations of GenBasis $\left(1^{n}, 1^{m}, q\right)$. For each $i>t^{*}+1$ (if any) and $b \in\{0,1\}$, generate $\mathbf{A}_{i}^{(b)} \in \mathbb{Z}_{q}^{n \times m}$ uniformly at random. 
$\mathcal{S}$ gives to $\mathcal{A}$ the master public key $m p k=\left(\mathbf{A}_{0},\left\{\mathbf{A}_{j}^{(b)}\right\}, \mathbf{y}, d\right)$, the encapsulation $\left(\mathbf{b}^{*}, p\right)$, and the key bit $k$.

Then $\mathcal{S}$ answers each secret-key query on an identity $i d$ that is not a prefix of (or equal to) $i d^{*}$ as follows:

- If $t=|i d| \leq t^{*}$, then let $i \geq 1$ be the first position at which $i d_{i} \neq i d_{i}^{*}$. Answer the query with $\left(\mathbf{S}_{i d}, \mathbf{e}_{i d}\right)$, which are computed by

$$
\begin{aligned}
& \mathbf{S}_{i d} \leftarrow \operatorname{RandBasis}\left(\operatorname{ExtBasis}\left(\mathbf{S}_{i}, \mathbf{A}_{i d}\right), s_{t}\right) \\
& \mathbf{e}_{i d} \leftarrow \operatorname{SampleD}\left(\operatorname{ExtBasis}\left(\mathbf{S}_{i}, \mathbf{A}_{i d}\right), \mathbf{y}_{i d}, s_{t}\right) .
\end{aligned}
$$

- If $t=|i d|>t^{*}$, answer the query $\left(\mathbf{S}_{i d}, \mathbf{e}_{i d}\right)$, which are computed by

$$
\begin{aligned}
& \mathbf{S}_{i d} \leftarrow \text { RandBasis }\left(\operatorname{ExtBasis}\left(\mathbf{S}_{t^{*}+1}^{\left(i d_{t^{*}+1}\right)}, \mathbf{A}_{i d}\right), s_{t}\right) \\
& \mathbf{e}_{i d} \leftarrow \operatorname{SampleD}\left(\operatorname{ExtBasis}\left(\mathbf{S}_{t^{*}+1}^{\left(i d_{t^{*}+1}\right)}, \mathbf{A}_{i d}\right), \mathbf{y}_{i d}, s_{t}\right) .
\end{aligned}
$$

Finally, $\mathcal{S}$ outputs whatever bit $\mathcal{A}$ outputs.

We now analyze the reduction. First, observe that the master public key given to $\mathcal{A}$ is negligibly close to uniform (hence properly distributed), by hypothesis on KEM and by Proposition 1. Next, one can check that secret-key queries are distributed as in the real attack (to within $\operatorname{negl}(n)$ statistical distance), by Lemma 4 (note that the GramSchmidt vectors of each basis $\mathbf{S}_{i}, \mathbf{S}_{t^{*}+1}^{(b)}$ are sufficiently short to invoke RandBasis and SampleD). Finally, the encapsulation $\left(\mathbf{b}^{*}, p\right)$ (for identity $\left.i d^{*}\right)$ and key bit $k$ are distributed as in the real attack, by the truncation property of KEM. Therefore, $\mathcal{S}$ 's overall advantage is within $\operatorname{negl}(n)$ of $\mathcal{A}$ 's advantage, as desired.

\subsection{Full Security in the Random Oracle Model}

To obtain a fully secure HIBE in the random oracle model we can use a generic transformation by Boneh and Boyen [8]. It starts from a selective-id secure HIBE and applies hash functions to the identities. The resulting HIBE is fully secure, in the random oracle model, losing roughly a factor of $Q_{H}^{d}$ in security, where $Q_{H}$ is the number of random oracle queries. Furthermore, the $\left\{\mathbf{A}_{j}^{(b)}\right\}$ component of the master public key may be omitted, because each $\mathbf{A}_{i d}$ can instead be constructed by querying the random oracle on, say, each prefix of the identity $i d$.

We now give a more efficient fully-secure HIBE scheme, ROHIBE, in the random oracle model. It can be seen as a generalization of the GPV IBE scheme [25]. Compared to the fully-secure scheme obtained by the generic transformation, the efficiency improvement stems from the fact that $\mathbf{y}$ from $p k_{i d}$ now also depends on the identity $i d$ (via a hash function $G$ ). This way the dimension of the lattice associated to $i d$ can be decreased. The scheme is again parametrized by a dimension $m=O(n \lg q)$ and the following parameters. For an identity at depth $i \geq 1$,

- $i \cdot m$ is the dimension of a lattice associated with the identity;

- $\widetilde{L_{i}}$ is an upper bound on the Gram-Schmidt lengths of its secret short basis;

- for $i \geq 1, s_{i}$ is the Gaussian parameter used to generate that secret basis, which must exceed $\widetilde{L_{j}} \cdot \omega(\sqrt{\log n})$ for all $j<i$. 
These parameters, along with the total depth $d$ of the hierarchy, determine the modulus $q$ and error distribution $\chi$ used in the cryptosystem. As before, we instantiate all the parameters after describing the scheme. Let $H:\{0,1\}^{*} \rightarrow \mathbb{Z}_{q}^{n \times m}$ and $G:\{0,1\}^{*} \rightarrow$ $\mathbb{Z}_{q}^{n}$ be hash functions.

- ROHIBE.Setup $(d)$ : This is the same as BTE.Setup $(d)$, except that we only generate $\mathbf{A}_{0}$ and $\mathbf{S}_{0}$. More precisely, using Proposition 1 , select $\mathbf{A}_{0} \in \mathbb{Z}_{q}^{n \times m}$ that is (negligibly close to) uniform and a basis $\mathbf{S}_{0}$ of $\Lambda^{\perp}\left(\mathbf{A}_{0}\right)$ such that $\|\widetilde{\mathbf{S}}\| \leq \widetilde{L_{0}}$. Output $m p k=\left(\mathbf{A}_{0}, d\right)$ and $m s k=\mathbf{S}_{0}$.

All the remaining algorithms implicitly take the master public key $m p k$ as an input. For an identity vector $i d$ of length $t \leq d$, we let

$$
\mathbf{A}_{i d}=\mathbf{A}_{0}\left\|\mathbf{A}_{1}\right\| \cdots \| \mathbf{A}_{t-1} \in \mathbb{Z}_{q}^{n \times t m}, \quad \mathbf{y}_{i d}=G(i d) \in \mathbb{Z}_{q}^{n},
$$

where $\mathbf{A}_{i}=H\left(i d_{1}, \ldots, i d_{i}\right) \in \mathbb{Z}_{q}^{n \times m}$. We let $p k_{i d}=\left(\mathbf{A}_{i d}, \mathbf{y}_{i d}\right)$ denote the KEM public key associated with identity vector $i d$.

- ROHIBE.Extract $\left(\mathbf{S}_{i d}, i d^{\prime}=i d \| \bar{i} d\right)$ : if $t^{\prime}=\left|i d^{\prime}\right|>d$, output $\perp$. Else, let $t=|i d|$ and $\bar{t}=|\bar{i} d|$, and choose

$$
\mathbf{S}_{i d^{\prime}} \leftarrow \operatorname{RandBasis}\left(\operatorname{ExtBasis}\left(\mathbf{S}_{i d}, \mathbf{A}_{i d^{\prime}}\right), s_{t^{\prime}}\right) .
$$

Sample $\mathbf{e}_{i d^{\prime}} \leftarrow D_{\Lambda_{\mathbf{y}_{i d^{\prime}}}^{\perp}\left(\mathbf{A}_{i d^{\prime}}\right), s_{t^{\prime}}}$ using SampleD(ExtBasis $\left.\left(\mathbf{S}_{i d}, \mathbf{A}_{i d^{\prime}}\right), \mathbf{y}_{i d^{\prime}}, s_{t^{\prime}}\right)$ and output $s k_{i d^{\prime}}=\left(\mathbf{S}_{i d^{\prime}}, \mathbf{e}_{i d^{\prime}}\right)$.

For technical reasons, we assume that the same $\mathbf{e}_{i d^{\prime}}$ is drawn every time this identity is used. This means that the actual algorithm should be stateless or use standard techniques like PRFs to get repeated randomness.

- ROHIBE.Encaps $(i d)$ : Output $(k, C) \leftarrow \operatorname{KEM}$.Encaps $\left(p k_{i d}\right)$.

- ROHIBE.Decaps $\left(s k_{i d}=\left(\mathbf{S}_{i d}, \mathbf{e}_{i d}\right), C\right)$ : Output $k \leftarrow \operatorname{KEM} . \operatorname{Decaps}\left(\mathbf{e}_{i d}, C\right)$.

Instantiating the parameters. A similar computation as in the last subsection shows that we can set

$$
\widetilde{L_{t}}=\widetilde{L_{0}} \cdot O(\sqrt{d \cdot n \lg q})^{t-1} \cdot \omega(\sqrt{\log n})^{t-1},
$$

with $\widetilde{L_{0}}=O(\sqrt{n \lg q})$. To ensure that the underlying KEM is complete (Lemma 5), we let $q \geq 4 s_{d} \cdot(d+1) m$ and $1 / \alpha=s_{d} \cdot \sqrt{(d+1) m} \cdot \omega(\sqrt{\log n})$. For any $d=\operatorname{poly}(n)$, invoking the worst-case to average-case reduction for LWE yields an underlying approximation factor of $n \cdot \tilde{O}(d \cdot \sqrt{n})^{d}$.

Theorem 3 (Security of ROHIBE). There exists a PPT oracle algorithm (a reduction) $\mathcal{S}$ attacking KEM (instantiated with dimension $d m$ and $q, \chi$ as in ROHIBE) such that, for any adversary $\mathcal{A}$ mounting an aid-ind-cpa attack on ROHIBE making $Q_{H}$ queries to the random oracle $H$ and $Q_{G}$ queries to the random oracle $G$,

$$
\mathbf{A d v}_{K E M}\left(\mathcal{S}^{\mathcal{A}}\right) \geq \mathbf{A d v}_{\text {ROHIBE }}^{\text {aid-ind-cpa }}(\mathcal{A}) /\left(d Q_{H}^{d-1} Q_{G}\right)-\operatorname{negl}(n) .
$$

Proof. Let $\mathcal{A}$ be an adversary mounting a aid-ind-cpa-attack on ROHIBE. We construct a reduction $\mathcal{S}$ attacking KEM. It is given a uniformly random public key $p k=(\mathbf{A}, \mathbf{y}) \in$ 
$\mathbb{Z}_{q}^{n \times d m} \times \mathbb{Z}_{q}^{n}$, an encapsulation $(\mathbf{b}, p) \in \mathbb{Z}_{q}^{d m} \times \mathbb{Z}_{q}$, and a bit $k$ which either is encapsulated by $(\mathbf{b}, p)$ or is uniform and independent; the goal of $\mathcal{S}$ is to determine which is the case.

Let $Q_{G}$ and $Q_{H}$ be the number or queries that $\mathcal{A}$ issues to $H$ and $G$, respectively. In our analysis, we will actually be more generous and let the adversary issue at most $d \cdot Q_{H}$ total queries, where it is allowed $Q_{H}$ queries to $H$ at each input length. To simplify the analysis, we also assume without loss of generality that (1) whenever $\mathcal{A}$ queries $H\left(i d_{1}, \ldots, i d_{i}\right)$, it has already issued the queries $H\left(i d_{1}, \ldots, i d_{j}\right)$ for $j<i$, and (2) that when $\mathcal{A}$ asks for $s k_{i d}$, it has already queried $H(i d)$ and $G(i d)$.

$\mathcal{S}$ simulates the attack on ROHIBE to $\mathcal{A}$ as follows. First, $\mathcal{S}$ produces a master public key $m p k$, encapsulated key, and some secret internal state as follows:

- Guess length of challenge identity and random oracle queries. Choose $t^{*} \leftarrow[d]$, a guess for the length of the challenge identity. Pick a vector $\mathbf{j}^{*}=\left(j_{1}^{*}, \ldots, j_{t^{*}-1}^{*}\right) \leftarrow$ $\left\{1, \ldots, Q_{H}\right\}^{t^{*}-1}$ and index $j \leftarrow\left\{1, \ldots, Q_{G}\right\}$.

- Parsing the KEM inputs. Parse $\mathbf{A}$ as $\mathbf{A}=\mathbf{A}_{0}\left\|\mathbf{A}_{1}\right\| \cdots \| \mathbf{A}_{d-1} \in \mathbb{Z}_{q}^{n \times d m}$ for $\mathbf{A}_{0} \in$ $\mathbb{Z}_{q}^{n \times m}$ and $\mathbf{A}_{i} \in \mathbb{Z}_{q}^{n \times m}$ for all $i \in[d-1]$. Similarly, truncate $\mathbf{b}$ to $\mathbf{b}^{*} \in \mathbb{Z}_{q}^{t^{*} m}$.

$\mathcal{S}$ gives to $\mathcal{A}$ the master public key $m p k=\left(\mathbf{A}_{0}, d\right)$. To simulate the attack game for $\mathcal{A}, \mathcal{S}$ must simulate oracle queries to $H$ and $G$, queries for user secret keys, and it must also generate the challenge encapsulation. To do this, it will maintain two lists, denoted $\mathcal{H}$ and $\mathcal{G}$, which are initialized to be empty and will store tuples of values. $\mathcal{S}$ processes queries as follows.

Queries to $H(\cdot)$. On $\mathcal{A}$ 's $j_{i}$-th distinct query $\left(i d_{j_{i}, 1}, \ldots, i d_{j_{i}, i}\right)$ to $H(\cdot)$ of length $i$, do the following: if $i \leq t^{*}-1$ and $j_{i}=j_{i}^{*}$, then return $\mathbf{A}_{i}$ (i.e., this branch undergoes undirected growth). Otherwise, if $i \geq t^{*}$ or $j_{i} \neq j_{i}^{*}$, run $\operatorname{GenBasis}\left(1^{n}, 1^{m}, q\right)$ to generate $\mathbf{A}_{i, j_{i}} \in \mathbb{Z}_{q}^{n \times m}$ with corresponding short basis $\mathbf{S}_{i, j_{i}}$ (i.e., this branch undergoes controlled growth). Store the tuple $\left(\left(i d_{j_{i}, 1}, \ldots, i d_{j_{i}, i}\right), \mathbf{A}_{i, j_{i}}, \mathbf{S}_{i, j_{i}}\right)$ in list $\mathcal{H}$, and return $\mathbf{A}_{i, j_{i}}$.

Queries to $G(\cdot)$. On $\mathcal{A}$ 's $j$-th distinct query $i d_{j}$ to $G(\cdot)$, do the following: if $j=j^{*}$ then return $\mathbf{y}$. (Recall that $\mathbf{y}$ was obtained from the KEM input.) Otherwise for $j \neq j^{*}$, sample $\mathbf{e}_{j} \leftarrow D_{\Lambda^{\perp}\left(\mathbb{Z}^{m}\right), s_{t}}$ (where $t$ is the depth of $i d_{j}$ ) and set $\mathbf{y}_{j}:=$ $\mathbf{A}_{\left(i d_{j, 1}, \ldots, i d_{j, t-1}\right)} \mathbf{e}_{j} \in \mathbb{Z}_{q}^{n}$. (Recall that we assumed $\mathcal{A}$ has already made all relevant queries to $H$ that in particular define $\mathbf{A}_{\left(i d_{j, 1}, \ldots, i d_{j, t-1}\right)}=H\left(i d_{j, 1}, \ldots, i d_{j, t-1}\right)$.) Return $\mathbf{y}_{j}$ and store $\left(i d_{j}, \mathbf{y}_{j}, \mathbf{e}_{j}\right)$ in list $\mathcal{G}$.

Queries to ROHIBE.Extract. When $\mathcal{A}$ asks for a user secret key for $i d=\left(i d_{1}, \ldots, i d_{t}\right)$, we again assume that $\mathcal{A}$ has already made all relevant queries to $G$ and $H$ that define $\mathbf{y}_{i d}$ and $\mathbf{A}_{i d}$. If, for one $i \in[t-1], \mathbf{A}_{i d_{i}}=H\left(i d_{1}, \ldots, i d_{i}\right)$ is contained in list $\mathcal{H}$, then $\mathcal{B}$ can compute a properly distributed short basis $\mathbf{S}_{i d}$ for $\mathbf{A}_{i d}$ by running RandBasis $\left(\right.$ ExtBasis $\left.\left(\mathbf{S}_{i, i d_{i}}, \mathbf{A}_{i d}\right), s_{t}\right)$, where $\mathbf{S}_{i, i d_{i}}$ is obtained from $\mathcal{H}$. If $\mathbf{y}_{i d}$ is contained in list $\mathcal{G}$, then $\mathcal{B}$ can retrieve a properly distributed vector $\mathbf{e}_{i d}$ from $\mathcal{G}$ satisfying $\mathbf{A}_{\left(i d_{1}, \ldots, i d_{t-1}\right)} \mathbf{e}_{i d}=\mathbf{y}_{i d}$. If the generation of $s k_{i d}=\left(\mathbf{B}_{i d}, \mathbf{e}_{i d}\right)$ was successful, then $\mathcal{B}$ returns $s k_{i d}$. In all other cases, $\mathcal{B}$ aborts (and returns a random bit).

Challenge query for $i d^{*}$. Let $t$ be the depth of $i d^{*}$. If $t \neq t^{*}$, or $G\left(i d^{*}\right) \neq \mathbf{y}$, or $H\left(i d_{1}^{*}, \ldots, i d_{i}^{*}\right) \neq \mathbf{A}_{i}$ (for one $1 \leq i \leq t^{*}-1$ ), then abort. Otherwise, return the encapsulation $\left(\mathbf{b}^{*}, p\right)$, and the key-bit $k$. 
$\mathcal{S}$ runs until $\mathcal{A}$ halts, and it outputs whatever bit $\mathcal{A}$ outputs.

It remains to analyze the reduction. The master public key given to $\mathcal{A}$ is negligibly close to uniform by the construction of KEM and Proposition 1 . By the same proposition, we have that oracle queries to $H$ are properly simulated, up to negligible statistical distance. Oracle responses for $G$ are negligibly far from uniform by Lemma 2 (4). It can also be verified using the truncation property of KEM that the challenge encapsulation is properly distributed, conditioned on the event that $\mathcal{S}$ does not abort. Thus all that remains to check is the probability that $\mathcal{S}$ aborts; this is at most $1 /\left(d Q_{G} Q_{H}^{d-1}\right)$. This completes the proof.

\subsection{Full Security in the Standard Model}

To achieve aid-ind-cca security in the standard model, we will essentially try to implement the random oracle from our scheme ROHIBE with a suitable hash function. We will employ a probabilistic argument, along the lines of [9]. Concretely, we will set up the public key such that in the simulation, we will know a short basis for $\mathbf{A}_{i d}$ with a certain probability. A sophisticated construction of the hash function will ensure that, to a certain degree, these probabilities (resp. the corresponding events) are independent. That is, even an adversary that adaptively asks for user secret keys will not manage to produce an identity $i d$ for which the simulation is guaranteed to know a trapdoor. In this case, a successful simulation will be possible.

Of course, we will have to take care that the event of a successful simulation is (at least approximately) independent of the adversary's view. To achieve independence, we will employ an "artificial abort" strategy similar to the one from [56].

Admissible hash functions. We give a variant of the definition from [9]. Let $\mathcal{H}=$ $\left\{\mathcal{H}_{n}\right\}$ be a collection of distributions of functions $H: \mathcal{C}_{n} \rightarrow \mathcal{D}_{n}=\{0,1\}^{\lambda}$. For $H \in \mathcal{H}_{n}, K \in\{0,1, \perp\}^{\lambda}$, and $x \in \mathcal{C}_{n}$, define

$$
F_{K, H}(x)=\left\{\begin{array}{ll}
\mathrm{B} & \text { if } \exists u \in\{1, \ldots, \lambda\}: t_{u}=K_{i} \\
\mathrm{R} & \text { if } \forall u \in\{1, \ldots, \lambda\}: t_{u} \neq K_{i}
\end{array} \quad \text { for }\left(t_{1}, \ldots, t_{\lambda}\right)=H(x) .\right.
$$

For $\mu \in\{0, \ldots, \lambda\}$, denote by $\mathcal{K}_{\mu}$ the uniform distribution on all keys $K \in\{0,1, \perp\}^{\lambda}$ with exactly $\mu$ non- $\perp$ components.

We say that $\mathcal{H}$ is $\Delta$-admissible (for $\Delta: \mathbb{N}^{2} \rightarrow \mathbb{R}$ ) if for every polynomial $Q=Q(n)$, there exists an efficiently computable function $\mu=\mu(n)$, and efficiently recognizable sets $\operatorname{bad}_{H} \subseteq\left(\mathcal{C}_{n}\right)^{*}\left(H \in \mathcal{H}_{n}\right)$, so that the following holds:

- For every PPT algorithm $\mathcal{C}$ that, on input a function $H \in \mathcal{H}_{n}$, outputs a vector $\mathbf{x} \in \mathcal{C}_{n}^{Q+1}$, the function

$$
\mathbf{A d v}_{\mathcal{H}}^{a d m}(\mathcal{C}):=\operatorname{Pr}\left[\mathbf{x} \in \operatorname{bad}_{H} \mid H \leftarrow \mathcal{H}_{n} ; \mathbf{x} \leftarrow \mathcal{C}(H)\right]
$$

is negligible in $n$.

- For every $H \in \mathcal{H}_{n}$ and every $\mathbf{x}=\left(x_{0}, \ldots, x_{Q}\right) \in \mathcal{C}_{n}^{Q+1} \backslash \operatorname{bad}_{H}$, we have that

$$
\operatorname{Pr}\left[F_{K, H}\left(x_{0}\right)=\mathrm{R} \wedge F_{K, H}\left(x_{1}\right)=\cdots=F_{K, H}\left(x_{Q}\right)=\mathrm{B}\right] \geq \Delta(n, Q),
$$

where the probability is over uniform $K \in \mathcal{K}_{\mu(n, Q)}$.

We say that $\mathcal{H}$ is admissible if $\mathcal{H}$ is admissible for some $\Delta$, such that $\Delta(n, Q)$ is significant for every polynomial $Q=Q(n)$. 
Difference to the definition of [9]. Note that our definition of admissibility is conceptually different from that of [9]. The reason for our change is that our definition is better suited for our purposes. Concretely, their definition is based upon indistinguishability from a (biased) random function. However, their construction only achieves asymptotic indistinguishability (i.e., negligible distinguishing success) when the "target" random function is constant. (In their notation, this corresponds to the case when $\gamma$ is negligible, so that $\operatorname{Pr}\left[F_{K, H}(x)=1\right]=1-\operatorname{negl}(n)$.) Such a function is not very useful for aymptotic purposes. In an asymptotic sense, their construction becomes only useful with parameters that cause the distinguishing advantage to become significant (but smaller than the inverse of a given polynomial). With that parameter choice, our definition allows for a conceptually simpler analysis. Namely, it separates certain negligible error probabilities ( of $\mathbf{x} \in \operatorname{bad}_{H}$ ) from significant, but purely combinatorial bounds on the probability of the "simulation-enabling" event

$$
\operatorname{good}:=\left[F_{K, H}\left(x_{0}\right)=\mathrm{R} \wedge F_{K, H}\left(x_{1}\right)=\cdots=F_{K, H}\left(x_{Q}\right)=\mathrm{B}\right] .
$$

Specifically, we can bound $\operatorname{Pr}$ [good] for every $\mathbf{x} \notin$ bad $_{H}$, which simplifies the artificial abort step below. Note that while the original analysis from [9] does not incorporate an artificial abort step, this actually would have been necessary to guarantee sufficient independence of (their version of) event good. This becomes an issue in [9, Claim 2], when the success probability of an adversary conditioned on good is related to its original (unconditioned) success probability.

Constructions. [9] show how to construct admissible hash functions from a given collision-resistant hash function family. Since collision-resistant hash functions can be built from the LWE problem (e.g., [5]), this does not entail extra assumptions in the encryption context. Specifically, for parameter choices as in [9, Section 5.3], we get a single hash function with output length $\lambda=O\left(k^{2+\varepsilon}\right.$ ) (for arbitrary $\varepsilon>0$ ) that is $\Delta$-admissible with $\Delta=\Theta\left(1 / Q^{2}\right) 5$

The scheme SMHIBE. Let $d \in \mathbb{N}$ denote the maximal depth of the HIBE, and fix a dimension $m$, as well as $\widetilde{L_{i}}, s_{i}$. Let $\mathcal{H}=\left(\mathcal{H}_{n}\right)_{n}$ be an admissible family of hash functions $H:\{0,1\}^{n} \rightarrow\{0,1\}^{\lambda}$.

SMHIBE.Setup $(d)$. Using Proposition 1, generate $\mathbf{A} \in \mathbb{Z}_{q}^{n \times m}$ and a corresponding short basis $\mathbf{S} \in \mathbb{Z}^{m \times m}$ with $\|\widetilde{\mathbf{S}}\| \leq \widetilde{L_{0}}$. Furthermore, sample uniformly and independently matrices $\mathbf{B}_{i, u, b} \in \mathbb{Z}_{q}^{n \times m}$ (for $1 \leq i \leq d, 1 \leq u \leq \lambda$ and $0 \leq b \leq 1$ ) and a vector $\mathbf{y} \in \mathbb{Z}_{q}^{n}$. Finally, choose $H_{1}, \ldots, H_{d} \leftarrow \mathcal{H}_{n}$. Return

$$
m p k=\left(\mathbf{A}, \mathbf{y},\left(\mathbf{B}_{i, u, b}\right)_{(i, u, b) \in[d] \times[\lambda] \times\{0,1\}},\left(H_{i}\right)_{i=1}^{d}\right), \quad m s k=(m p k, \mathbf{S}) .
$$

For an identity $i d=\left(i d_{1}, \ldots, i d_{\ell}\right)$ we define

$$
\begin{aligned}
\mathbf{A}_{i d} & :=\mathbf{A}\left\|\mathbf{A}_{1, i d_{1}}\right\| \ldots \| \mathbf{A}_{\ell, i d_{\ell}} \in \mathbb{Z}_{q}^{n \times(\lambda \ell+1) m} \\
\text { for } \quad \mathbf{A}_{i, i d_{i}} & :=\mathbf{B}_{i, 1, t_{1}}\|\ldots\| \mathbf{B}_{i, \lambda, t_{\lambda}} \in \mathbb{Z}_{q}^{n \times \lambda m}
\end{aligned}
$$

\footnotetext{
${ }^{5}$ In the notation of [9], we replace the output length $\beta_{H}$ of the original hash function with $k$, and bound the number $Q$ of hash function queries by $2^{k^{\varepsilon / 2}}$. Note that $Q$ will later correspond to the number of (online) user secret key queries, so we bound $Q$ by a comparatively small exponential function.
} 
where $\left(t_{1}, \ldots, t_{\lambda}\right):=H_{i}\left(i d_{i}\right) \in\{0,1\}^{\lambda}$. The user secret keys for an identity $i d$ will consist of a basis part $\mathbf{S}_{i d}$ for $\Lambda^{\perp}\left(\mathbf{A}_{i d}\right)$ and a syndrome part $\mathbf{e}_{i d}$ satisfying $\mathbf{A}_{i d} \mathbf{e}_{i d}=\mathbf{y}$. For brevity, we will write $i d \mid \ell:=\left(i d_{1}, \ldots, i d_{\ell}\right)$ for $\ell \leq|i d|$.

SMHIBE.Extract $(m s k, i d)$ : This algorithm computes a user secret key $\left(\mathbf{S}_{i d}, \mathbf{e}_{i d}\right)$ for $i d=\left(i d_{1}, \ldots, i d_{\ell}\right)$, where $\mathbf{S}_{i d} \leftarrow \operatorname{RandBasis}\left(\operatorname{ExtBasis}\left(\mathbf{A}_{i d}, \mathbf{S}_{\varepsilon}\right), s_{\ell}\right)$ is a basis for $\Lambda^{\perp}\left(\mathbf{A}_{i d}\right)$ and $\mathbf{e}_{i d} \leftarrow$ SampleD $\left(\right.$ ExtBasis $\left.\left(\mathbf{A}_{i d}, \mathbf{S}_{\varepsilon}\right), \mathbf{y}_{i d}, s_{\ell}\right)$ is distributed according to $D_{\mathbb{Z}^{(\lambda \ell+1) m}, s_{\ell}}$ conditioned on $\mathbf{A}_{i d} \mathbf{e}_{i d}=\mathbf{y}_{i d}$.

SMHIBE.HIBEDel $\left(u s k_{i d \mid \ell-1}, i d\right)$ : The delegation algorithm derives a user secret key for an identity $i d=\left(i d_{1}, \ldots, i d_{\ell}\right)(1 \leq \ell \leq d)$ given a user secret key for $i d \mid \ell-1$ which contains a basis $\mathbf{S}_{i d \mid \ell-1}$ for $\Lambda^{\perp}\left(\mathbf{A}_{i d \mid \ell-1}\right)$ with $\left\|\widetilde{\mathbf{S}}_{i d \mid \ell-1}\right\| \leq L(\ell-1)$. (We note that the short vector $\mathbf{e}_{i d \mid \ell-1}$ is not needed for delegation.) Note that $\mathbf{A}_{i d}=\mathbf{A}\left\|\mathbf{A}_{1, i d_{1}}\right\| \cdots\left\|\mathbf{A}_{\ell, i d_{\ell}}=\mathbf{A}_{1, i d \mid \ell-1}\right\| \mathbf{A}_{\ell, i d_{\ell}} \in \mathbb{Z}_{q}^{n \times(\lambda \ell+1) m}$. To compute the basis part, run $\mathbf{S}_{i d} \leftarrow \operatorname{RandBasis}\left(\operatorname{ExtBasis}\left(\mathbf{A}_{i d}, \mathbf{S}_{i d \mid \ell-1}\right), s_{\ell}\right)$. Note that since $\ell$ is constant,

$$
\begin{aligned}
& L(\ell)=L(\ell-1) \cdot \sqrt{\lambda m} \cdot \omega(\sqrt{\log \lambda m}) \\
& \geq\left\|\widetilde{\mathbf{S}}_{i d \mid \ell-1}\right\| \cdot \sqrt{(\lambda \ell+1) m} \cdot \omega(\sqrt{\log (\lambda \ell+1) m}) .
\end{aligned}
$$

The syndrome part of the user secret key is computed as

$$
\mathbf{e}_{i d} \leftarrow \operatorname{SampleD}\left(\operatorname{ExtBasis}\left(\mathbf{A}_{i d}, \mathbf{S}_{i d \mid \ell-1}\right), \mathbf{y}, s_{\ell}\right) .
$$

By Lemma 4, the user secret key $u s k_{i d}=\left(\mathbf{S}_{i d}, \mathbf{e}_{i d}\right)$ has a distribution that is statistically close to the one computed by Extract.

SMHIBE.Encaps $(i d, b)$ : Output $C=(k, \mathbf{p}) \leftarrow \operatorname{KEM} . \operatorname{Encaps}\left(p k=\left(\mathbf{A}_{i d}, \mathbf{y}\right)\right)$.

SMHIBE.Decaps $\left(s k_{i d},\left(\mathbf{S}_{i d}, \mathbf{e}_{i d}\right), C\right)$ : Output $k \leftarrow \operatorname{KEM} . \operatorname{Decaps}\left(\mathbf{e}_{i d}, C\right)$.

The scheme's correctness is inherited by that of KEM.

Security of SMHIBE. We now formally state security of our construction. If the hash function $\mathcal{H}$ is admissible, then we can prove the scheme aid-ind-cpa secure. Unfortunately, we only know constructions of admissible hash functions that require $\lambda=n^{2+\varepsilon}$ so the resulting scheme is not very practical.

Theorem 4. Assume an adversary $\mathcal{A}$ on SM-HIBE's aid-ind-cpa security that makes at most $Q(n)$ user secret key queries. Then, for every polynomial $S=S(n)$, there exists an $\operatorname{LWE}_{q, \chi}$-distinguisher $\mathcal{D}$ and an adversary $\mathcal{C}$ on $\mathcal{H}$ 's admissibility such that

$$
\operatorname{Adv}_{\mathrm{SM}-\mathrm{HIBE}}^{\text {aid-ind-cpa }}(\mathcal{A}) \leq d \cdot \mathbf{A d v}_{\mathcal{H}}^{a d m}(\mathcal{C})+\frac{\mathbf{A d v}_{\mathrm{LWE}_{q, \chi}}(\mathcal{D})}{\Delta(n, Q)^{d}}+\frac{1}{S(n)}+\operatorname{negl}(n) .
$$

Here, the running time of $\mathcal{C}$ is roughly that of the aid-ind-cpa experiment with $\mathcal{A}$, and the running time of $\mathcal{D}$ is roughly that of the aid-ind-cpa experiment with $\mathcal{A}$ plus $O\left(n^{2} Q S / \Delta^{d}\right)$ steps.

Note that for the admissible hash function from [9], $\Delta(n, Q)^{d}=\Theta\left(1 / Q^{2 d}\right)$ is significant. Since $S$ in Theorem 4 is arbitrary, we obtain:

Corollary 1 (SM-HIBE is aid-ind-cpa secure). If $\mathcal{H}$ is admissible, and if the $\mathrm{LWE}_{q, \chi}$ problem is hard, then SM-HIBE is CPA secure.

We defer a proof of Theorem 4 to the full version. 


\section{Acknowledgments}

We thank the anonymous Eurocrypt reviewers for their helpful comments, and for pointing out a small error in an earlier formulation of RandBasis.

\section{References}

[1] Abdalla, M., Bellare, M., Catalano, D., Kiltz, E., Kohno, T., Lange, T., Malone-Lee, J., Neven, G., Paillier, P., Shi, H.: Searchable encryption revisited: Consistency properties, relation to anonymous IBE, and extensions. J. Cryptology 21(3), 350-391 (2008); Preliminary version in CRYPTO 2005

[2] Agrawal, S., Boneh, D., Boyen, X.: Efficient lattice (H)IBE in the standard model. In: EUROCRYPT 2010 (to appear, 2010)

[3] Agrawal, S., Boyen, X.: Identity-based encryption from lattices in the standard model (July 2009) (manuscript)

[4] Ajtai, M.: Generating hard instances of the short basis problem. In: Wiedermann, J., Van Emde Boas, P., Nielsen, M. (eds.) ICALP 1999. LNCS, vol. 1644, pp. 1-9. Springer, Heidelberg (1999)

[5] Ajtai, M.: Generating hard instances of lattice problems. Quaderni di Matematica 13, 1-32 (2004); Preliminary version in STOC 1996

[6] Alwen, J., Peikert, C.: Generating shorter bases for hard random lattices. In: STACS, pp. 75-86 (2009)

[7] Bellare, M., Boldyreva, A., Desai, A., Pointcheval, D.: Key-privacy in public-key encryption. In: Boyd, C. (ed.) ASIACRYPT 2001. LNCS, vol. 2248, pp. 566-582. Springer, Heidelberg (2001)

[8] Boneh, D., Boyen, X.: Efficient selective-ID secure identity-based encryption without random oracles. In: Cachin, C., Camenisch, J.L. (eds.) EUROCRYPT 2004. LNCS, vol. 3027, pp. 223-238. Springer, Heidelberg (2004)

[9] Boneh, D., Boyen, X.: Secure identity based encryption without random oracles. In: Franklin, M. (ed.) CRYPTO 2004. LNCS, vol. 3152, pp. 443-459. Springer, Heidelberg (2004)

[10] Boneh, D., Canetti, R., Halevi, S., Katz, J.: Chosen-ciphertext security from identity-based encryption. SIAM J. Comput. 36(5), 1301-1328 (2007)

[11] Boneh, D., Di Crescenzo, G., Ostrovsky, R., Persiano, G.: Public key encryption with keyword search. In: Cachin, C., Camenisch, J.L. (eds.) EUROCRYPT 2004. LNCS, vol. 3027, pp. 506-522. Springer, Heidelberg (2004)

[12] Boneh, D., Franklin, M.K.: Identity-based encryption from the weil pairing. SIAM J. Comput. 32(3), 586-615 (2003); Preliminary version in CRYPTO 2001

[13] Boneh, D., Gentry, C., Hamburg, M.: Space-efficient identity based encryption without pairings. In: FOCS, pp. 647-657 (2007)

[14] Boyen, X., Niçoises, L., Trapdoors, V.: A Framework for Fully Secure Short Signatures and more. In: PKC 2010 (to appear, 2010)

[15] Boyen, X., Waters, B.: Anonymous hierarchical identity-based encryption (without random oracles). In: Dwork, C. (ed.) CRYPTO 2006. LNCS, vol. 4117, pp. 290-307. Springer, Heidelberg (2006)

[16] Canetti, R., Halevi, S., Katz, J.: A forward-secure public-key encryption scheme. J. Cryptology 20(3), 265-294 (2007); Preliminary version in EUROCRYPT 2003

[17] Cash, D., Hofheinz, D., Kiltz, E.: How to delegate a lattice basis. Cryptology ePrint Archive, Report 2009/351 (July 2009), http: / / eprint.iacr.org/

[18] Cocks, C.: An identity based encryption scheme based on quadratic residues. In: IMA Int. Conf., pp. 360-363 (2001) 
[19] Cramer, R., Shoup, V.: Signature schemes based on the strong RSA assumption. ACM Trans. Inf. Syst. Secur. 3(3), 161-185 (2000); Preliminary version in CCS 1999

[20] Di Crescenzo, G., Saraswat, V.: Public key encryption with searchable keywords based on Jacobi symbols. In: Srinathan, K., Rangan, C.P., Yung, M. (eds.) INDOCRYPT 2007. LNCS, vol. 4859, pp. 282-296. Springer, Heidelberg (2007)

[21] Dodis, Y., Fazio, N.: Public key broadcast encryption for stateless receivers. In: ACM Workshop on Digital Rights Management, pp. 61-80 (2002)

[22] Gennaro, R., Halevi, S., Rabin, T.: Secure hash-and-sign signatures without the random oracle. In: Stern, J. (ed.) EUROCRYPT 1999. LNCS, vol. 1592, pp. 123-139. Springer, Heidelberg (1999)

[23] Gentry, C.: Practical identity-based encryption without random oracles. In: Vaudenay, S. (ed.) EUROCRYPT 2006. LNCS, vol. 4004, pp. 445-464. Springer, Heidelberg (2006)

[24] Gentry, C., Halevi, S.: Hierarchical identity based encryption with polynomially many levels. In: Reingold, O. (ed.) TCC 2009. LNCS, vol. 5444, pp. 437-456. Springer, Heidelberg (2009)

[25] Gentry, C., Peikert, C., Vaikuntanathan, V.: Trapdoors for hard lattices and new cryptographic constructions. In: STOC, pp. 197-206 (2008)

[26] Gentry, C., Silverberg, A.: Hierarchical ID-based cryptography. In: Zheng, Y. (ed.) ASIACRYPT 2002. LNCS, vol. 2501, pp. 548-566. Springer, Heidelberg (2002)

[27] Goldreich, O., Goldwasser, S., Halevi, S.: Public-key cryptosystems from lattice reduction problems. In: Kaliski Jr., B.S. (ed.) CRYPTO 1997. LNCS, vol. 1294, pp. 112-131. Springer, Heidelberg (1997)

[28] Goldwasser, S., Micali, S., Rivest, R.L.: A digital signature scheme secure against adaptive chosen-message attacks. SIAM J. Comput. 17(2), 281-308 (1988); Preliminary version in FOCS 1984

[29] Hoffstein, J., Howgrave-Graham, N., Pipher, J., Silverman, J.H., Whyte, W.: NTRUSIGN: Digital signatures using the NTRU lattice. In: Joye, M. (ed.) CT-RSA 2003. LNCS, vol. 2612, pp. 122-140. Springer, Heidelberg (2003)

[30] Hoffstein, J., Pipher, J., Silverman, J.H.: NTRU: A ring-based public key cryptosystem. In: Cohen, H. (ed.) ANTS 1996. LNCS, vol. 1122, pp. 267-288. Springer, Heidelberg (1996)

[31] Hohenberger, S., Waters, B.: Realizing hash-and-sign signatures under standard assumptions. In: Joux, A. (ed.) EUROCRYPT 2009. LNCS, vol. 5479, pp. 333-350. Springer, Heidelberg (2009)

[32] Hohenberger, S., Waters, B.: Short and stateless signatures from the rsa assumption. In: Halevi, S. (ed.) CRYPTO 2009. LNCS, vol. 5677, pp. 654-670. Springer, Heidelberg (2009)

[33] Horwitz, J., Lynn, B.: Toward hierarchical identity-based encryption. In: Knudsen, L.R. (ed.) EUROCRYPT 2002. LNCS, vol. 2332, pp. 466-481. Springer, Heidelberg (2002)

[34] Krawczyk, H., Rabin, T.: Chameleon signatures. In: NDSS (2000)

[35] Leurent, G., Nguyen, P.Q.: How risky is the random-oracle model? In: Halevi, S. (ed.) CRYPTO 2009. LNCS, vol. 5677, pp. 445-464. Springer, Heidelberg (2009)

[36] Lyubashevsky, V., Micciancio, D.: Generalized compact knapsacks are collision resistant. In: Bugliesi, M., Preneel, B., Sassone, V., Wegener, I. (eds.) ICALP 2006, Part II. LNCS, vol. 4052, pp. 144-155. Springer, Heidelberg (2006)

[37] Lyubashevsky, V., Micciancio, D.: Asymptotically efficient lattice-based digital signatures. In: Canetti, R. (ed.) TCC 2008. LNCS, vol. 4948, pp. 37-54. Springer, Heidelberg (2008)

[38] Lyubashevsky, V., Peikert, C., Regev, O.: On ideal lattices and learning with errors over rings. In: EUROCRYPT (to appear, 2010)

[39] Micciancio, D.: Generalized compact knapsacks, cyclic lattices, and efficient one-way functions. Computational Complexity 16(4), 365-411 (2007); Preliminary version in FOCS 2002 
[40] Micciancio, D., Goldwasser, S.: Complexity of Lattice Problems: a cryptographic perspective. The Kluwer International Series in Engineering and Computer Science, vol. 671. Kluwer Academic Publishers, Boston (2002)

[41] Micciancio, D., Regev, O.: Worst-case to average-case reductions based on Gaussian measures. SIAM J. Comput. 37(1), 267-302 (2007); Preliminary version in FOCS 2004

[42] Micciancio, D., Warinschi, B.: A linear space algorithm for computing the Hermite normal form. In: ISSAC, pp. 231-236 (2001)

[43] Naor, M., Yung, M.: Universal one-way hash functions and their cryptographic applications. In: STOC, pp. 33-43 (1989)

[44] Peikert, C.: Bonsai trees (or, arboriculture in lattice-based cryptography). Cryptology ePrint Archive, Report 2009/359 (July 2009), http: / / eprint.iacr.org/

[45] Peikert, C.: Public-key cryptosystems from the worst-case shortest vector problem. In: STOC, pp. 333-342 (2009)

[46] Peikert, C.: An efficient and parallel Gaussian sampler for lattices (2010) (manuscript)

[47] Peikert, C., Rosen, A.: Efficient collision-resistant hashing from worst-case assumptions on cyclic lattices. In: Halevi, S., Rabin, T. (eds.) TCC 2006. LNCS, vol. 3876, pp. 145-166. Springer, Heidelberg (2006)

[48] Peikert, C., Rosen, A.: Lattices that admit logarithmic worst-case to average-case connection factors. In: STOC, pp. 478-487 (2007)

[49] Peikert, C., Vaikuntanathan, V., Waters, B.: A framework for efficient and composable oblivious transfer. In: Wagner, D. (ed.) CRYPTO 2008. LNCS, vol. 5157, pp. 554-571. Springer, Heidelberg (2008)

[50] Rabin, M.O.: Digitalized signatures and public-key functions as intractable as factorization. Technical Report MIT/LCS/TR-212, MIT Laboratory for Computer Science (1979)

[51] Regev, O.: On lattices, learning with errors, random linear codes, and cryptography. J. ACM 56(6) (2009); Preliminary version in STOC 2005

[52] Rückert, M.: Strongly unforgeable signatures and hierarchical identity-based signatures from lattices without random oracles. In: PQCrypto (to appear, 2010)

[53] Shamir, A.: Identity-based cryptosystems and signature schemes. In: Blakely, G.R., Chaum, D. (eds.) CRYPTO 1984. LNCS, vol. 196, pp. 47-53. Springer, Heidelberg (1985)

[54] Shamir, A., Tauman, Y.: Improved online/offline signature schemes. In: Kilian, J. (ed.) CRYPTO 2001. LNCS, vol. 2139, pp. 355-367. Springer, Heidelberg (2001)

[55] Stehlé, D., Steinfeld, R., Tanaka, K., Xagawa, K.: Efficient public key encryption based on ideal lattices. In: Matsui, M. (ed.) ASIACRYPT 2009. LNCS, vol. 5912, pp. 617-635. Springer, Heidelberg (2009)

[56] Waters, B.: Efficient identity-based encryption without random oracles. In: Cramer, R. (ed.) EUROCRYPT 2005. LNCS, vol. 3494, pp. 114-127. Springer, Heidelberg (2005)

[57] Waters, B.: Dual system encryption: Realizing fully secure IBE and HIBE under simple assumptions. In: Halevi, S. (ed.) CRYPTO 2009. LNCS, vol. 5677, pp. 619-636. Springer, Heidelberg (2009)

[58] Yao, D., Fazio, N., Dodis, Y., Lysyanskaya, A.: ID-based encryption for complex hierarchies with applications to forward security and broadcast encryption. In: ACM Conference on Computer and Communications Security, pp. 354-363 (2004) 